\title{
TRANSVERSALITY IN THE SETTING OF HYPERBOLIC AND PARABOLIC MAPS
}

\author{
GENADI LEVIN, WEIXIAO SHEN AND SEBASTIAN VAN STRIEN
}

Dedicated to Lawrence Zalcman.

\begin{abstract}
In this paper we consider families of holomorphic maps defined on subsets of the complex plane, and show that the technique developed in 24 to treat unfolding of critical relations can also be used to deal with cases where the critical orbit converges to a hyperbolic attracting or a parabolic periodic orbit. As before this result applies to rather general families of maps, such as polynomial-like mappings, provided some lifting property holds. Our Main Theorem states that either the multiplier of a hyperbolic attracting periodic orbit depends univalently on the parameter and bifurcations at parabolic periodic points are generic, or one has persistency of periodic orbits with a fixed multiplier.
\end{abstract}

\section{INTRODUCTION}

When studying families of maps defined on an open subset of the complex plane, it is useful to have certain transversality properties. For example, do multipliers of attracting periodic points depend univalently on the parameter and do parabolic periodic points undergo generic bifurcations? Building on a method developed in 24] we establish such transversality results in a very general setting. The conclusion of our Main Theorem states that one has either such transversality or persistency of periodic points with the same multiplier holds.

The key assumption in our Main Theorem is a so-called lifting property defined in $\$ 2.2$. It turns out that this assumption is applicable in rather general settings, including families of maps with an infinite number of singular values, such as polynomial-like mappings and also maps with essential singularities.

Although the Main Theorem applies to complex maps, let us first mention applications to certain families of real maps. For example, consider the periodic doubling cascade associated to the family $f_{\lambda}=\lambda x(1-x), x \in[0,1]$ and $\lambda \in[0,4]$. It is well-known that the multiplier $\kappa(\lambda)$ of attracting periodic orbit decreases in $\lambda$ diffeomorphically in each interval for which $\kappa(\lambda) \in[-1,1)$ and that one has generic bifurcations when $\kappa(\lambda)= \pm 1$. An application of our result is that the same conclusion holds for families of the form $f_{\lambda}(x)=\lambda f(x)$ and similarly for $g_{c}(z)=g(z)+c$ where $f$ and $g$ are rather general interval maps. An important feature of our method is that we obtain information about the sign of the derivative of $\kappa^{\prime}$, namely that $\kappa^{\prime}>0$, see $\$ 8$.

The main aim of this paper is to obtain results which apply to maps which are defined only locally, e.g., having essential singularities. It turns out that many more classical results can be recovered also. Before stating our results more formally, let us discuss previous results and the approach that is used in this paper.

The study of the dependence of a multiplier on parameters goes back to DouadyHubbard [10, 11], who obtained the celebrated result (using Sullivan's quasiconformal surgery) that the multiplier map $\kappa$ uniformizes hyperbolic components of the Mandelbrot set. Milnor [33] generalized this result to spaces of hyperbolic polynomials and Rees [35] to the space of degree two rational maps. Douady-Hubbard theorem is equivalent to the local claim that $\kappa^{\prime} \neq 0$ whenever $|\kappa|<1$. In [22] and independently in [15] the latter local result is generalized to spaces of polynomials and rational maps. 
In [22], a polynomial or a rational function $f$ is considered which has $r$ cycles $O_{i}$ with corresponding multipliers $\kappa_{i} \in \overline{\mathbb{D}}=\{|\kappa| \leq 1\}$ such that if, for some $i, \kappa_{i}=1$ then $O_{i}$ is not degenerate and if $\kappa_{i}=0$ then $O_{i}$ contains a single critical point which is simple. Now consider a local space $\Lambda$, containing $f$, of functions with a constant number of different critical values and with constant multiplicities at the critical points. Then the multipliers of those $r$ cycles contribute $r$ independent parameters in the coordinate system of $\Lambda$, see [22, Theorems 2 and 6] for precise statements. The proof, which is a development of [19], [27], 20], [21], see also [28], relies on a non-trivial identity [22, Theorems 1 and 5] between, on the one hand, a specific function associated to a given cycle $O$ of $f$, and on the other hand, the gradient $\nabla \kappa$ of the multiplier of this cycle as a function of the coordinates of $\Lambda$. If $\kappa=1$, a modification is needed by replacing the gradient by

$$
\lim (1-\kappa(g)) \nabla \kappa(g)
$$

as $g \rightarrow f$ along $g$ having a cycle near $O$ with multiplier $\kappa(g) \neq 1$. A calculation as in Lemma 2.8 shows that the limit is equal to

$$
\left.\left(f^{q}\right)^{\prime \prime}(a) \nabla g^{q}\right|_{g=f}(a)
$$

(independently on $a \in O$ ). In particular, if $f(z)=z^{2}+c_{1}$ so that $\Lambda=\left\{g_{w}=z^{2}+w\right\}$, it gives

$$
\left.\frac{d}{d w} g_{w}^{q}\left(a_{k}\right)\right|_{w=c_{1}} \neq 0
$$

This recovers Douady-Hubbard's result [1] that every primitive component of the Mandelbrot set has a simple cusp. The Main Theorem of the present paper, which can be found on page 5. is a far reaching generalization of this fundamental fact to suitable families of local maps. The paper [22] contains also a detailed historical account.

In turn, in Epstein's work [15, Proposition 20] the above result is proven in the case when all $\kappa_{i} \in \overline{\mathbb{D}} \backslash\{0,1\}$ under the assumption that all critical points of $f$ are simple and $r$ is maximal (i.e., $r=\operatorname{deg} f-1$ for polynomials and $r=2 \operatorname{deg} f-2$ for rational functions). Epstein's work [15], see also [16], is a development after his earlier work [13], [14], is coordinate free and can also be used to prove transversality when there are critical relations. The techniques build on an approach pioneered by Thurston. In Thurston's approach, when $f$ is a globally defined holomorphic map, $P$ is a finite $f$-forward invariant set containing the postcritical set and the Thurston map $\sigma_{f}$ : Teich $(\widehat{\mathbb{C}} \backslash P) \rightarrow$ Teich $(\widehat{\mathbb{C}} \backslash P)$ is defined by pulling back an almost holomorphic structure. It turns out that $\sigma_{f}$ is contracting, see [12, Corollary 3.4]. In Thurston's result on the topological realisation of rational maps, Douady \& Hubbard [12] use that the dual of the derivative of the Thurston map $\sigma_{f}$ is equal to the Thurston pushforward operator $f_{*}$ which acts on the space of quadratic differentials. Epstein's approach is to exploit the injectivity of $f_{*}-i d$. One of the innovations in [15] is the introduction of the space Teichmüller deformation space $\operatorname{Def}_{A}^{B}(f)$ which can be used in a wide range of settings.

There were other approaches for critically finite rational maps: Sullivan's pull-back argument [38, 29, 30]. Milnor and Thurston's [34] and Tsujii's [39, 40, work. Their initial purpose was to prove the monotonicity of entropy for the real quadratic family. All these and previously discussed methods break down if the map is not globally defined, see [25] for details. In [24, 25] we develop a method which goes back to Milnor and Thurston's and Tsujii's approaches in allowing us to deal with maps which are locally defined. Milnor and Thurston associated to the space of quadratic maps and a given combinatorial data on a periodic orbit $P \ni 0$ of $f_{c_{1}}$ of period $q$ where $f_{c}(z) \equiv z^{2}+c$, a map which assigns to a $q$ tuple of points a new $q$ tuple of points,

$$
F:\left(z_{1}, \ldots z_{q}\right) \mapsto\left(\hat{z}_{1}, \ldots, \hat{z}_{q}\right)
$$

where $\hat{z}_{q}=0$ and $f_{z_{1}}\left(\hat{z}_{i}\right)=z_{i+1 \bmod q}$. Since $F$ is many-valued, Milnor and Thurston considered a lift $\tilde{F}$ of this map to the universal cover and apply Teichmüller theory to show that $\tilde{F}$ is strictly contracting in the Teichmuller metric of the universal cover. 
In [24, 25] we bypass this 'global' approach by rephrasing it locally. This is done in the set-up of so-called (locally defined) marked maps (and their local deformations) and replacing $F$ by an (non-linear) operation which associates to a holomorphic motion $h_{\lambda}$ of a finite set $P$ another holomorphic motion $\hat{h}_{\lambda}$ of $P$ that we call its lift. The lifting property is an assumption that sequences of successive lifts of holomorphic motions are compact. In [24], 25] we considered the case that the set $P$ is finite and prove that assuming the lifting property either some 'critical relation' persists along some non-trivial manifold in parameter space or one has transversality, i.e. the 'critical relation' unfolds transversally. In [24], 25] we also prove the lifting property for many interesting families of maps. An important feature of our method is that for real maps we obtain positive transversality originated in [39, 40]: some determinant is not only non-zero but has positive sign.

Here we consider maps with a non-finite 'postcritical set' $P$, namely locally defined maps with an attracting or a parabolic periodic orbit. The results of this paper do not aim to replicate the results mentioned previously, so we only consider the case of maps with a single attracting or parabolic periodic orbit. The proof follows the same strategy as in [24, 25]. Although we don't use the transfer operator which is defined in [24], 25] as the linearisation of the lift operator (and is infinitely dimensional if $P$ is infinite), the proof here has a strong 'flavor' of it, see the beginning of Sect 4 and Appendix A. As mentioned, the maps we consider are allowed to have essential singularities.

For other results for transversality in the setting of polynomial, rational or finite type maps (which have at most a finite number of singular values), see [3, 7, 9, 11, 17, 18, 23, 26, 37.

1.1. Organisation of this paper. In $\$ 2$ we will state the results of this paper, in $\$ 3$ we will give an outline of the three steps used in the proof, which will be given in 46 . Applications to complex and real families will be then given in $\$ 7,8$.

Acknowledgments. We thank Mitsu Shishikura and Michel Zinsmeister for very fruitful discussions. We also thank Alex Eremenko for inspiring us to write this paper, by posing the question which we answer in Theorem 2.3. We also would like to thank the anonymous referee. The authors acknowledge Adam Epstein for pointing out an error/typo at the very end of the proof of Lemma 4.1 in the Arxiv version of this paper. This work was started when GL visited Imperial College (London) and continued during his visit at the Institute of Mathematics of PAN (Warsaw). He thanks both Institutions for their generous hospitality. GL acknowledges the support of ISF grant No. 1226/17, WS acknowledges the support of NSFC grant No. 11731003, and SvS acknowledges the support of ERC AdG Grant No. 339523 RGDD.

\section{Statement of Results}

2.1. Marked maps and holomorphic deformations. Let $U$ an open subset of $\mathbb{C}$ and $g: U \rightarrow \mathbb{C}$ be holomorphic on $U$. Assume that $c_{1} \in U$. Then we say that $g$ is a marked map w.r.t. $c_{1}$ if $c_{n}=g^{n-1}\left(c_{1}\right)$ is well-defined for all $n \geq 1$ and $\bar{P} \subset U$ where $P=\left\{c_{n}\right\}_{n=1}^{\infty}$. We say that $(g, G)_{W}$ is a local holomorphic deformation of $g$ if:

(1) $W$ is an open connected subset of $\mathbb{C}$ containing $c_{1}$;

(2) $G:(w, z) \in W \times U \mapsto G_{w}(z) \in \mathbb{C}$ is a holomorphic map such that $G_{c_{1}}=g$;

(3) $D G_{w}(z) \neq 0$ for all $z \in U, w \in W$.

Here and later on $D=\frac{\partial}{\partial z}, D^{k}=\frac{\partial^{k}}{\partial z^{k}}$. Note that $c_{1}$ plays the role of a special point in the dynamical space $U$, but it is also a special point in the parameter space $W$.

Example 2.1. Let $g: U \rightarrow \mathbb{C}$ be a marked map w.r.t. $c_{1}$. Moreover, let $W=\mathbb{C}$ and $G: W \times U \rightarrow \mathbb{C}$ be defined by $G(w, z)=G_{w}(z)=g(z)+\left(w-c_{1}\right)$. Then $(g, G)_{W}$ is a local holomorphic deformation of $g$. 
2.2. Holomorphic motions and the lifting property. Recall that $h_{\lambda}$ is a holomorphic motion of a set $X \subset \mathbb{C}$ over $(\Lambda, 0)$ where $\Lambda$ is a domain in $\mathbb{C}$ which contains 0 , if $h_{\lambda}: X \rightarrow \mathbb{C}$ satisfies: (i) $h_{0}(x)=x$, for all $x \in X$, (ii) $h_{\lambda}(x) \neq h_{\lambda}(y)$ whenever $x \neq y$ and $\lambda \in \Lambda, x, y \in X$ and (iii) $\Lambda \ni \lambda \mapsto h_{\lambda}(z)$ is holomorphic. Quite often we will consider the special case where $\Lambda$ is equal to $\mathbb{D}_{r}:=B(0, r)$ where $B(a, r):=\{w \in \mathbb{C}:|w-a|<r\}$.

Let $K$ be a bounded set such that $P \subset K \subset \bar{K} \subset U$ and $g(K) \subset K$. The following notions of the lift and the lifting property were introduced and studied in [24, 25] (in the case of a finite set $K$ ):

Definition 2.2. Given a holomorphic motion $h_{\lambda}$ of $K$ over $(\Lambda, 0)$, we say that a holomorphic motion $\hat{h}_{\lambda}$ of $K$ over $\left(\Lambda_{0}, 0\right)$ where $\Lambda_{0} \subset \Lambda$, is a lift of $h_{\lambda}$ for $(g, G)_{W}$ if for all $\lambda \in \Lambda_{0}$ and $x \in K$,

$$
G_{h_{\lambda}\left(c_{1}\right)}\left(\hat{h}_{\lambda}(x)\right)=h_{\lambda}(g(x)) .
$$

We say that $(g, G)_{W}$ has the lifting property for the set $K$ if the following holds: Given a holomorphic motion $h_{\lambda}^{(0)}$ of $K$ over $(\Lambda, 0)$, there exist $\varepsilon>0$ and holomorphic motions $h_{\lambda}^{(n)}, n=1,2,, \ldots$ of $K$ over $\left(\mathbb{D}_{\varepsilon}, 0\right)$ such that for each $n \geq 0$,

(1) $h_{\lambda}^{(n)}\left(c_{1}\right) \in W$ for each $\lambda \in \mathbb{D}_{\varepsilon}$;

(2) $h_{\lambda}^{(n+1)}$ is the lift of $h_{\lambda}^{(n)}$ over $\left(\mathbb{D}_{\varepsilon}, 0\right)$ for $(g, G)_{W}$,

(3) there exists $M>0$ such that $\left|h_{\lambda}^{(n)}(x)\right| \leq M$ for all $x \in K, \lambda \in \mathbb{D}_{\varepsilon}$ and $n \geq 0$.

The next lemma and the subsequent remark clarify this notion:

Lemma 2.3. Given any holomorphic motion $h_{\lambda}$ of $K$ over $\left(\mathbb{D}_{\epsilon_{0}}, 0\right)$, there is a sequence of holomorphic motions $h_{\lambda}^{(n)}, n \geq 0$, of $K$ where $h_{\lambda}^{(0)}=h_{\lambda}$ and $h_{\lambda}^{(n)}$ is the lift of $h_{\lambda}^{(n-1)}$ over $\left(\mathbb{D}_{\varepsilon_{n}}, 0\right)$ with some maximal $\varepsilon_{n}>0$.

Proof. It is enough to show that given a holomorphic motion $h_{\lambda}$ of $K$ over $\left(\mathbb{D}_{\epsilon_{0}}, 0\right)$, there exists $\epsilon_{1}>0$ (which depends on $\epsilon_{0}>0$ and $h_{\lambda}$ ) such that the lift $\hat{h}_{\lambda}$ of $h_{\lambda}$ for the set $K$ exists over $\mathbb{D}_{\epsilon_{1}}$. Indeed, as $\bar{K}$ is compact it follows from the definition of local holomorphic deformation that there are a finite open covering $\left\{B\left(x_{j}, r_{j}\right)\right\}$ of $K$ and $\epsilon_{1}>0$ such that, for $\lambda \in \mathbb{D}_{\epsilon_{1}}$ the following hold: $h_{\lambda}\left(c_{1}\right) \in W$, for each $j$ the map $G_{h_{\lambda}\left(c_{1}\right)}$ is injective on $B\left(x_{j}, 2 r_{j}\right)$ and $h_{\lambda}(g(y)) \in G_{h_{\lambda}\left(c_{1}\right)}\left(B\left(x_{j}, 2 r_{j}\right)\right)$ for $y \in B\left(x_{j}, r_{j}\right)$. Then define $\hat{h}_{\lambda}(y):=G_{h_{\lambda}\left(c_{1}\right)}^{-1}\left(h_{\lambda}(g(y))\right.$ for $y \in B\left(x_{j}, r_{j}\right)$ where $G_{h_{\lambda}\left(c_{1}\right)}^{-1}$ is the inverse map to $\left.G_{h_{\lambda}\left(c_{1}\right)}\right|_{B\left(x_{j}, 2 r_{j}\right)}$.

Remark 2.4. The lifting property means that for any holomorphic motion $h_{\lambda}$ of $K$ over $(\Lambda, 0)$, there is $\epsilon>0$ such that in the previous lemma $\varepsilon_{n}>\varepsilon$ holds for all $n \geq 0$ and such that the family $\left\{h_{\lambda}^{(n)}(x)\right\}$ is uniformly founded on $\mathbb{D}_{\varepsilon} \times K$.

Remark 2.5. Note that if $K_{1} \subset K_{2}$ are two forward invariant sets then the lifting property for $K_{2}$ implies the lifting property for $K_{1}$ (this follows from Slodkowski's generalised lambda lemma [36] and also [2]).

In [24, 25], we studied the case when the 'postcritical set' $P$ is finite and showed that if this lifting property holds then, under suitable circumstances, critical relations unfold transversally. The present paper considers the case where $P$ is an infinite orbit converging to an attracting or neutral periodic orbit and the main result shows that, again, the lifting property implies transversality.

2.3. The statement of Main Theorem. In this paper we will study marked maps $g$ such that $P=\left\{c_{n}\right\}_{n=1}^{\infty}$ is an infinite orbit of $g$ so that $c_{n}$ converges to a periodic orbit $\mathcal{O}=\left\{a_{0}, a_{1}, \ldots, a_{q-1}\right\}$ and consider a holomorphic deformation $(g, G)_{W}$ of $g$. The main theorem of this paper shows that if $(g, G)_{W}$ satisfies the lifting property, then the orbit $\mathcal{O}$ depends 'transversally' on the parameter $w$ (in a sense made precise below). 
As usual, we say that $\mathcal{O}$ is hyperbolic attracting if $\kappa:=D g^{q}\left(a_{0}\right) \in \mathbb{D} \backslash\{0\}$. We say that $\mathcal{O}$ is non-degenerate parabolic if there exists $l, p \in \mathbb{Z}, p \geq 1,(l, p)=1$ such that $\kappa=e^{2 \pi i l / p}$ and $D^{p+1} g^{p q}\left(a_{0}\right) \neq 0$. Let

$$
Q(z):=\left.\frac{d}{d w} G_{w}^{q}(z)\right|_{w=c_{1}},
$$

which is a holomorphic function defined in a neighborhood of $\mathcal{O} \cup P$.

Example 2.6. If $\kappa=1$ then non-degeneracy means that $D^{2} g^{q}\left(a_{0}\right) \neq 0$. If, moreover, $Q\left(a_{0}\right) \neq 0$ then the Taylor expansion of $G_{w}^{q}(z)$ at $z=a_{0}, w=c_{1}$ is given by $G_{w}^{q}(z)=$ $z+\alpha\left(z-a_{0}\right)^{2}+\beta\left(w-c_{1}\right)+$ h.o.t. with $\alpha, \beta \neq 0$. Our Main Theorem states that a certain lifting property implies that either $Q\left(a_{0}\right) \neq 0$ or that the parabolic periodic point persists for all parameters $w$ near $c_{1}$.

We will also use the following subset of the basin of $\mathcal{O}$ :

$$
\Omega_{r}:=\left\{z \in U: \sup _{n=0}^{\infty} d\left(f^{n}(z), \mathcal{O}\right) \leq r, \text { and } \lim _{n \rightarrow \infty} d\left(f^{n}(z), \mathcal{O}\right)=0\right\} \text { where } r>0 .
$$

In the attracting case, $\bigcup B\left(a_{j}, \rho\right) \subset \Omega_{r} \subset \bigcup \overline{B\left(a_{j}, r\right)}$ whenever $r$ is small enough and some even smaller $\rho>0$. In the parabolic case, the Leau-Fatou Flower Theorem (see Lemma B.1 in Appendix B) tells us that $\left(\bigcup B\left(a_{j}, \rho\right)\right) \backslash \Omega_{r}$, even though non-empty, is located in a very thin region near the repelling directions.

Main Theorem. Assume that $\mathcal{O}$ is either hyperbolic attracting or non-degenerate parabolic and that $(g, G)_{W}$ satisfies the lifting property for $P_{r_{0}}=P \cup \Omega_{r_{0}}$ for some $r_{0}>0$. Then one has

(1) either the following transversality property:

$$
D^{2} g^{q}\left(a_{0}\right) Q\left(a_{0}\right) \neq Q^{\prime}\left(a_{0}\right)(\kappa-1) ;
$$

(2) or persistency of periodic points with the same multiplier holds: there is a neighborhood $W_{1}$ of $c_{1}$ and holomorphic functions $a_{j}(w)$ defined in $W_{1}$ with $a_{j}\left(c_{1}\right)=a_{j}$ such that for each $w \in W_{1}, G_{w}^{q}\left(a_{j}(w)\right)=a_{j}(w)$ and $D G_{w}^{q}\left(a_{0}(w)\right)=\kappa$ is constant.

In this paper we will not check whether a particular family of maps satisfies the lifting property, but refer to the results proved in the second part of [24, 25] where the following is shown (with obvious changes in the proof):

Lemma 2.7. Let $f_{w}, U$ be one of the following.

- $f_{w}(z)=f(z)+w, w \in \mathbb{C}$ and $U=D \backslash\{0\}$ with $f \in \mathcal{F}$ defined in $\$ 2.5$,

- $f_{w}(z)=w f(z), w \in \mathbb{C} \backslash\{0\}$, with $f \in \mathcal{E} \cup \mathcal{E}_{0}$ defined in $\$ 2.6$ and $U=D \backslash f^{-1}(1)$ for $f \in \mathcal{E}, U=D \backslash \pm f^{-1}(1)$ for $f \in \mathcal{E}_{0}$.

Let $c_{1}$ be attracted to either hyperbolic attracting or parabolic periodic orbit of $f_{c_{1}}$ and $W_{1}$ is a neighborhood of $c_{1}$. Then $g=\left.f_{c_{1}}\right|_{U}$ is a marked map, $G_{w}=\left.f_{w}\right|_{U}, w \in W_{1}$ is a holomorphic deformation of $g$ and $(g, G)_{W}$ satisfies the lifting property for any $P_{r_{0}} \subset U$.

The above classes of families include families of maps with an essential singularity, such as $G_{w}(z)=b e^{-1 /|z|^{\ell}}+w$ for real non-zero $z$. A lifting property also holds within the spaces of rational and polynomial maps, see [25, Appendix C].

\subsection{Clarifying the transversality condition $(2.4)$ and the non-degeneracy con-} dition.

Lemma 2.8. (1) If $\kappa=1$ then (2.4) implies

$$
Q\left(a_{j}\right) \neq 0 \text { for all } j=0,1, \ldots, q-1 .
$$


(2) If $\kappa \neq 1$ then there exists a holomorphic function $w \mapsto a_{0}(w)$ for $w$ near $c_{1}$ so that $a_{0}(w)$ is a fixed point of $G_{w}^{q}$ for all $w$ near $c_{1}$ and so that $a_{0}\left(c_{1}\right)=a_{0}$. Defining $\kappa(w)=D G_{w}^{q}\left(a_{0}(w)\right)$ we have that 2.4) implies

$$
\kappa^{\prime}\left(c_{1}\right)=\frac{D^{2} g^{q}\left(a_{0}\right) Q\left(a_{0}\right)-Q^{\prime}\left(a_{0}\right)(\kappa-1)}{1-\kappa} \neq 0 .
$$

Proof. If $\kappa=1$, then $(2.4)$ is reduced to $Q\left(a_{0}\right) \neq 0$, which is equivalent to $Q\left(a_{j}\right) \neq 0$ for all $j=0,1, \ldots, q-1$. If $\kappa \neq 1$ then by the Implicit Function Theorem, there exists holomorphic functions $a_{j}(w)$, defined near $c_{1}$ such that $a_{j}\left(c_{1}\right)=a_{j}$ and $G_{w}\left(a_{j}(w)\right)=$ $a_{j+1}(w)$ for all $0 \leq j<q$, where $a_{q}(w)=a_{0}(w)$. Let $\kappa(w)=D G_{w}^{q}\left(a_{0}(w)\right)$. To see that (2.6) holds, let $\mathcal{G}(w, z)=G_{w}^{q}(z)$. Then

$$
\mathcal{G}\left(w, a_{j}(w)\right)=a_{j}(w), \frac{\partial \mathcal{G}}{\partial z}\left(w, a_{j}(w)\right)=\kappa(w),
$$

for each $j$. Differentiating and evaluating at $w=c_{1}$, we obtain

$$
Q\left(a_{j}\right)=\left(1-D g^{q}\left(a_{j}\right)\right) a_{j}^{\prime}\left(c_{1}\right), Q^{\prime}\left(a_{j}\right)+D^{2} g^{q}\left(a_{j}\right) a_{j}^{\prime}\left(c_{1}\right)=\kappa^{\prime}\left(c_{1}\right) .
$$

Thus the equality in 2.6 holds. The inequality in 2.6 is equivalent to (2.4).

Remark 2.9. In the parabolic case, the non-degeneracy condition is necessary as shown by the following example. Let $G_{w}(z)=w \sin z$. Choose $a_{0} \in(\pi / 2,3 \pi / 2)$ so that $\tan a_{0}=$ $-a_{0}$ and let $w_{0}=1 / \cos a_{0}, g=G_{w_{0}}$. Then $\mathcal{O}=\left\{a_{0},-a_{0}\right\}$ is a cycle of $g$ of period 2 with $g^{\prime}\left(a_{0}\right)=g^{\prime}\left(a_{1}\right)=1$. This parabolic cycle attracts both critical values $w_{0}$ and $-w_{0}$ of $g$ and is degenerate. On the other hand,

$$
Q\left(a_{0}\right)=\left.\frac{d}{d w} G_{w}^{2}\left(a_{0}\right)\right|_{w=w_{0}}=\sin \left(-a_{0}\right)+D g\left(-a_{0}\right) \sin \left(a_{0}\right)=0 .
$$

Note that at the parameter $w_{0}=1 / \cos \left(a_{0}\right) \approx-2.26$ the family of maps $G_{w}(x)=w \sin (x)$, $w \in \mathbb{R}$ undergoes a pitchfork bifurcation, where the attracting period two orbit of this map for $w \in\left(w_{0}, w_{0}+\epsilon\right)$ becomes for $w \in\left(w_{0}-\epsilon, w_{0}\right)$ a repelling two orbit and splits-off two new periodic orbits, both of which are attracting, see Figure 1 in the last section.

2.5. Applications to transversality within additive complex families. Let us start by complex families of the form $f_{c}(z)=f(z)+c, c \in \mathbb{C}$. Let $\mathcal{F}$ denote the collection of holomorphic maps $f: D \rightarrow V$, where

(1) $D$ is a bounded open set in $\mathbb{C}$ with $0 \in \bar{D}$;

(2) $V$ is a bounded open set in $\mathbb{C}$;

(3) $f: D \backslash\{0\} \rightarrow V \backslash\{0\}$ is an un-branched covering;

(4) The following separation property holds: $V \supset B(0 ; \operatorname{diam}(D)) \supset D$.

Theorem 2.1. Let $f \in \mathcal{F}$ and let $G_{w}(z)=f(z)+w$. Suppose that $c_{1} \in \bar{D}$ is such that $g=G_{c_{1}}$ has an attracting or parabolic cycle $\mathcal{O}=\left\{a_{0}, \cdots, a_{q-1}\right\}$ with multiplier $\kappa \neq 0$. Then $c_{n}=g^{n-1}\left(c_{1}\right)$ is well defined and converges to $\mathcal{O}$ as $n \rightarrow \infty$ and the following transversality holds:

$$
\kappa^{\prime}\left(c_{1}\right) \neq 0 \text { if } \kappa \neq 1 \text { and } Q\left(a_{j}\right) \neq 0 \text { for } a_{j} \in \mathcal{O} \text { if } \kappa=1 .
$$

Example 2.10. The conclusion of the above theorem applies for example for the following families:

- $f_{c}(z)=z^{d}+c, c \in \mathbb{C}$ and where $d \geq 2$ is an integer.

- $f_{c}(x)=b e^{-1 /|x|^{\ell}}+c$ where $\ell \geq 1, b>2(e \ell)^{1 / \ell}$ are fixed and $c \in D$. Here $D=$ $U^{-} \cup U^{+}, U^{-}=-U^{+}, U^{ \pm}$are disjoint topological disks symmetric w.r.t. the real axis and $\{0\}=\overline{U^{+}} \cap \overline{U^{-}}$. Furthermore, there is $R>1$ such that $f_{0}: D \rightarrow \mathbb{D}_{R} \backslash\{0\}$ is an unbranched covering, $D \subset \mathbb{D}_{R}$, and $U^{+} \cap \mathbb{R} \supset(0, \beta]$ where $\beta>0$ is so that the map $f_{-\beta}$ has the Chebysheb combinatorics: $f_{-\beta}(\beta)=\beta$.

Remark 2.11. When $G_{c}$ is a real family, the sign of $\kappa^{\prime}$ and $Q\left(a_{0}\right)$ is given in Section 8 , see also Appendix A. 
Remark 2.12. For the quadratic family $G_{c}(z)=z^{2}+c$ the inequalities in (2.7) were already known. The inequality $\kappa^{\prime}\left(c_{1}\right) \neq 0$ for $c_{1}$ so that $G_{c_{1}}$ has a hyperbolic attracting periodic point was established in [11. When $c_{1}$ is real and $G_{c_{1}}$ has either a hyperbolic attracting or a parabolic periodic point with multiplier +1 , the signs for $\kappa^{\prime}\left(c_{1}\right)$ and $Q\left(a_{0}\right)$ were also already known, see for example [32, Lemma 4.5].

2.6. Applications to transversality within multiplicative complex families. To state our next theorem, we say that $v$ is an asymptotic value of a holomorphic map $f: D \rightarrow \mathbb{C}$ if there exists a path $\gamma:[0,1) \rightarrow D$ so that $\gamma(t) \rightarrow \partial D$ and $f(\gamma(t)) \rightarrow v$ as $t \uparrow 1$. We say that $v$ is a singular value if it is a critical value or an asymptotic value. Let us next consider families of the form $f_{w}(z)=w f(z)$ where $f: D \rightarrow V$ is a holomorphic map such that:

(a) $D, V$ are open sets which are symmetric w.r.t. the real line so that $f(D)=V$;

(b) Let $I=D \cap \mathbb{R}$ then there exists $c>0$ so that $I \cup\{c\}$ is a (bounded or unbounded) open interval and $0 \in \bar{I}, c \in \operatorname{int}(\bar{I})$. Moreover, $f$ extends continuously to $\bar{I}$ so that $f(I) \subset \mathbb{R}$ and $\lim _{z \in D, z \rightarrow 0} f(z)=0$.

(c) Let $D_{+}$be the component of $D$ which contains $I \cap(c, \infty)$, where $D_{+}$might be equal to $D$. Then $u \in D \backslash\{0\}$ and $v \in D_{+} \backslash\{0\}, v \neq u$, implies $u / v \in V$.

Let $\mathcal{E}$ be the class of maps which satisfy $(a),(b),(c)$ and assumption $(d)$ :

(d) $f: D \rightarrow V$ has no singular values in $V \backslash\{0,1\}$ and $c>0$ is minimal such that $f$ has a positive local maximum at $c$ and $f(c)=1$.

Similarly let $\mathcal{E}_{o}$ be the class of maps which satisfy $(a),(b),(c)$ and assumption $(e)$ :

(e) $f$ is odd, $f: D \rightarrow V$ has no singular values in $V \backslash\{0, \pm 1\}$ and $c>0$ is minimal such that $f$ has a positive local maximum at $c$ and $f(c)=1$.

The class $\mathcal{F}$ was introduced in [24] and classes $\mathcal{E}, \mathcal{E}_{o}$ in [24, 25] and include maps for which $V$ or $D$ are bounded sets.

Theorem 2.2. Let $f: D \rightarrow V$ be holomorphic map from $\mathcal{E} \cup \mathcal{E}_{o}$ and define $g=c_{1} \cdot f: D \rightarrow$ $c_{1} \cdot V$ where we assume that $c_{1} \in D_{+} \backslash\{0\}$. Assume that $g$ has a hyperbolic attracting or a parabolic cycle $\mathcal{O}=\left\{a_{0}, \cdots, a_{q-1}\right\} \subset D \backslash\{0\}$ with multiplier $\kappa$. Take $G_{w}(z)=w f(z)$ where $w \in W:=D_{+} \backslash\{0\}$. Then at $w=c_{1}$, one of the following holds:

- transversality holds:

$$
\kappa^{\prime}\left(c_{1}\right) \neq 0 \text { if } \kappa \neq 1 \text { and } Q\left(a_{j}\right) \neq 0 \text { for } a_{j} \in \mathcal{O} \text { if } \kappa=1,
$$

- $f \in \mathcal{E}_{o}$ and $\mathcal{O}$ is symmetric with respect to the origin.

Example 2.13. The conclusion of the previous theorem applies for example to the following families:

- $f_{b}(z)=b z(1-z), b \in \mathbb{C} \backslash\{0\}$,

- $f_{b}(z)=b \exp (z)(1-\exp (z)), b \in \mathbb{C} \backslash\{0\}$,

- $f_{b}(z)=b[\sin (z)]^{2}, b \in \mathbb{C} \backslash\{0\}$,

- $f_{b}(z)=b \sin (z), b \in \mathbb{C} \backslash\{0\}$,

- $f_{b}(z)=b f(z)$ where $f$ is the unimodal map $f:[0,1] \rightarrow[0,1]$ defined by

$$
f(x)=\exp \left(2^{\ell}\right)\left(-\exp \left(-1 /|x-1 / 2|^{\ell}\right)+\exp \left(-2^{\ell}\right)\right)
$$

satisfying $f(0)=f(1)=0, f(1 / 2)=1$ which has a flat critical point at $c=1 / 2$. Here $\ell \geq \ell_{0}$ where $\ell_{0}$ is chosen sufficiently large. This implies that $f$ has an extension $f: D \rightarrow V$ which is in $\mathcal{E}_{0}$, where $V$ is a punctured bounded disc and $D$ consists of two components $D_{-} \cup D_{+}$. Here we assume that the parameter $b \in D_{+}$.

Remark 2.14. The classes $\mathcal{E}$ and $\mathcal{E}_{o}$ both contain maps for which the set of singular values has infinite cardinality. 
2.7. Periodic points do not disappear after they are born. For real maps, additional arguments allow us to obtain the sign of $\kappa^{\prime}\left(c_{1}\right)$ and $Q\left(a_{0}\right)$.

Each $f \in \mathcal{E} \cup \mathcal{E}_{o}$ defines naturally a unimodal map $f: J:=(0, b) \rightarrow \mathbb{R}$ where

$$
b=\sup \left\{b^{\prime} \in I: b^{\prime}>0 \text { and } f(x)>0 \text { for } x \in\left(0, b^{\prime}\right)\right\} .
$$

We denote by $\mathcal{E}_{u}$ and $\mathcal{E}_{o, u}$ the collection of unimodal maps obtained in this way from maps in $\mathcal{E}$ and $\mathcal{E}_{o}$ respectively. Recall that $c$ is the turning point of $f$ in $J$.

We denote by $\mathcal{F}_{u}^{+}$(resp. $\mathcal{F}_{u}^{-}$) the collection of $C^{1}$ unimodal maps $f: J \rightarrow \mathbb{R}$, where $J \ni 0$ is an open interval, such that $f \mid J \backslash\{0\}$ allows an extension to a map $F: D \rightarrow V$ in $\mathcal{F}$ with $J \backslash\{0\}=(D \cap \mathbb{R}) \backslash\{0\}$ and such that $f$ has a maximum (resp. minimum) at 0 . Put $c=\{0\}$.

Theorem 2.3. Consider a family of unimodal maps $f_{t}$ satisfying one of the following:

(i) $f_{t}=f+t$ with $f \in \mathcal{F}_{u}^{+}$and $t \in J$;

(ii) $f_{t}=t \cdot f$ with $f \in \mathcal{E}_{u} \cup \mathcal{E}_{o, u}$ and $t \in J$.

Suppose $f_{t_{*}}, t_{*}>c$, has a period cycle $\mathcal{O}$, then for any $t \in J$ with $t \geq t_{*}, f_{t}$ has a periodic cycle $\mathcal{O}_{t}$ of the same period such that $\mathcal{O}_{t}$ depends on $t$ continuously and $\mathcal{O}_{t_{*}}=\mathcal{O}$.

Similarly for

(iii) $f_{t}=f+t$ with $f \in \mathcal{F}_{u}^{-}$and $t \in J$,

if $f_{t_{*}}, t_{*}<c$, has a period cycle $\mathcal{O}$, then for any $t \in J$ with $t \leq t_{*}, f_{t}$ has a periodic cycle $\mathcal{O}_{t}$ of the same period such that $\mathcal{O}_{t}$ depends on $t$ continuously and $\mathcal{O}_{t_{*}}=\mathcal{O}$.

\section{Outline of the Proof of the Main Theorems}

The setting in this paper (and it's purpose) is similar to that in 24], 25] except there the case where the postcritical set is finite is considered. Here we will follow the same strategy in the proof as in that paper. So let us recall the main steps in the proof of Theorem 2.1 of [24] or the Main Theorem of [25] :

(A) Assume that transversality fails. Then there exists a holomorphic motion $h_{\lambda}$ of $P$ over $\left(\mathbb{D}_{\epsilon}, 0\right)$ with the speed $v$ at $\lambda=0$ :

$$
\left.\frac{d h_{\lambda}\left(c_{n}\right)}{d \lambda}\right|_{\lambda=0}=v\left(c_{n}\right)=\left.\frac{d}{d w} G_{w}^{n-1}(w)\right|_{w=c_{1}} \text { for } n=1,2, \cdots .
$$

(B) Let $h_{\lambda}^{(0)}=h_{\lambda}$ and $h_{\lambda}^{(k+1)}$ be the lift of $h_{\lambda}^{(k)}$ for $k=0,1,2, \cdots$. By the lifting property, all holomorphic motions $h_{\lambda}^{(k)}$ of $P$ are defined over $\left(\mathbb{D}_{\hat{\epsilon}}, 0\right)$ for some $\hat{\epsilon}>0$ and are uniformly bounded. Moreover, by (A), all $h_{\lambda}^{(k)}$ are asymptotically invariant of order $m=1$, i.e.,

$$
h_{\lambda}^{(k+1)}\left(c_{n}\right)-h_{\lambda}^{(k)}\left(c_{n}\right)=O\left(\lambda^{m+1}\right)
$$

with $m=1$. Consider averages

$$
\hat{h}_{\lambda}^{(k)}(z)=\frac{1}{k} \sum_{i=0}^{k-1} h_{\lambda}^{(i)}(z),
$$

$k=1,2, \cdots$ and let $\hat{h}_{\lambda}$ be a limit map of $\hat{h}_{\lambda}^{(k)}$ along a subsequence. Then:

(B1) $\hat{h}_{\lambda}$ is again a holomorphic motion of $P$ over (perhaps, smaller) disk,

(B2) $\hat{h}_{\lambda}$ is asymptotically invariant of order $m+1=2$.

(B3) Repeating the procedure, we find that for every $m=1,2, \ldots$ there is a holomorphic motion which is asymptotically invariant of order $m$.

(C) Finally, the (B3) yields that the 'critical relation' persists for all $w$ in a manifold containing $c_{1}$ of dimension $>0$.

When $P$ is a finite set, steps (A) and (B1) are straightforward. In the current set-up this can be also made to work, as is shown in this paper, but sometimes with considerable technical efforts. Moreover, we need to require the lifting property to be satisfied not 
only on the postcritical set $P$ but also on a bigger set which is a local basin of attraction of either hyperbolic or parabolic cycle.

Definition 3.1. A holomorphic motion $h_{\lambda}$ of $P_{r_{0}}:=P \cup \Omega_{r_{0}}$ over $\left(\mathbb{D}_{\varepsilon}, 0\right)$ is called admissible if for each $\lambda \in \mathbb{D}_{\varepsilon}, z \mapsto h_{\lambda}(z)$ is holomorphic in the interior of $\Omega_{r_{0}}$. It is called asymptotically invariant of order $m$ if for each $z \in P$,

$$
\widehat{h}_{\lambda}(z)-h_{\lambda}(z)=O\left(\lambda^{m+1}\right) \text { as } \lambda \rightarrow 0
$$

where $\widehat{h}_{\lambda}$ is the lift of $h_{\lambda}$.

If $h_{\lambda}$ is asymptotically invariant of order $m$, then: (1) its lift $\widehat{h}_{\lambda}$ is asymptotically invariant of order $m$, too, and (2) $G_{h_{\lambda}\left(c_{1}\right)}\left(h_{\lambda}(x)\right)=h_{\lambda}(g(x))+O\left(\lambda^{m+1}\right), x \in P$. See [25] for details.

The proof of the Main Theorem is broken into the following three steps.

Theorem A. Assume transversality fails, so assume equality holds in (2.4). Then there exists an admissible holomorphic motion $H_{\lambda}$ of the set $P_{r_{0}}$ over $\left(\mathbb{D}_{\epsilon}, 0\right)$ for some $\epsilon>0$ such that

$$
\left.\frac{d H_{\lambda}}{d \lambda}\right|_{\lambda=0}\left(c_{n}\right)=v\left(c_{n}\right), n=1,2, \cdots,
$$

where $v\left(c_{n}\right)=\left.\frac{d}{d w} G_{w}^{n-1}(w)\right|_{w=c_{1}}$. In particular, the holomorphic motion is asymptotically invariant of order 1 .

Theorem B. For any $m \geq 1$, if there is an admissible holomorphic motion $h_{\lambda}$ of the set $P_{r_{0}}$ over some $\mathbb{D}_{\varepsilon}$ which is asymptotically invariant of order $m$, then there is an admissible holomorphic motion $\widetilde{h}_{\lambda}$ of the set $P_{r_{0}}$ over some $\mathbb{D}_{\varepsilon^{\prime}}$ which is asymptotically invariant of order $m+1$ such that

$$
\widetilde{h}_{\lambda}(z)-h_{\lambda}(z)=O\left(\lambda^{m+1}\right) \text { as } \lambda \rightarrow 0, \text { for any } z \in P .
$$

Theorem C. Suppose for any $m \geq 1$, there is an admissible holomorphic motion $h_{\lambda, m}$ of $\bar{P}_{r_{0}}$ over $\left(\mathbb{D}_{\varepsilon_{m}}, 0\right)$ for some $\varepsilon_{m}>0$ such that

$$
\left.\frac{d}{d \lambda} h_{\lambda, m}\left(c_{1}\right)\right|_{\lambda=0}=1
$$

and $h_{\lambda, m}$ is asymptotically invariant of order $m$. Then the second alternative of the Main Theorem holds.

Proof of the Main Theorem. Assume that the transversality condition fails. Then by Theorems A and B, we obtain a sequence of admissible holomorphic motions $h_{\lambda, m}$ of $\bar{P}_{r_{0}}$ satisfying the assumption of Theorem C. Thus the second alternative of the Main Theorem holds.

Theorems A and B will be proved in Sect 4 and 5 respectively, where the hyperbolic case is much easier and will be done first. Theorem $\mathrm{C}$ will be proved in Sect 6 .

3.1. How to construct admissible holomorphic motions. We end this section with the following lemma which is useful in constructing admissible holomorphic motions.

Lemma 3.2. Let $h_{\lambda}$ be a holomorphic motion of $P_{r_{0}}$ over $\left(\mathbb{D}_{\varepsilon}, 0\right)$ for some $\varepsilon>0$ which is asymptotically invariant of order $m$. Assume that for each $K_{0}>1$, there is a g-invariant open set $W \subset P_{r_{0}}$ such that

(1) $z \mapsto h_{\lambda}(z)$ is $K_{0}-q c$ in $W$ for all $\lambda \in \mathbb{D}_{\varepsilon}$;

(2) $\Omega_{r_{0}} \subset \bigcup_{n=0}^{\infty} g^{-n}(W)$.

Then there exists an admissible holomorphic motion $\check{h}_{\lambda}$ of $P_{r_{0}}$ over $\left(\mathbb{D}_{\varepsilon^{\prime}}, 0\right)$ for some $\varepsilon^{\prime}>0$ such that

$$
h_{\lambda}\left(c_{k}\right)-\check{h}_{\lambda}\left(c_{k}\right)=O\left(\lambda^{m+1}\right) \text { for each } k \geq 1 \text {. }
$$


Proof. By the lifting property, restricting to a smaller disk $\mathbb{D}_{\varepsilon^{\prime}}$, the holomorphic motion $h_{\lambda}$ allows successive lifts $h_{\lambda}^{(n)}$ of $P_{r_{0}}$ over $\mathbb{D}_{\varepsilon^{\prime}}$. By compactness of holomorphic motions, there exists $n_{k} \rightarrow \infty$, such that $h_{\lambda}^{\left(n_{k}\right)}$ converges to a holomorphic motion $\check{h}_{\lambda}$ of $P_{r_{0}}$ over $\mathbb{D}_{\varepsilon^{\prime}}$ locally uniformly.

For each $k \geq 1$, by asymptotic invariance of $h_{\lambda}, h_{\lambda}^{(n)}\left(c_{k}\right)-h_{\lambda}\left(c_{k}\right)=O\left(\lambda^{m+1}\right)$, hence (3.2) holds.

Let us prove that $z \mapsto \check{h}_{\lambda}(z)$ is holomorphic in $\Omega_{r_{0}}^{o}$. To this end, it suffices to show that $\check{h}_{\lambda}$ is $K_{0}$-qc in $\Omega_{r_{0}}^{o}$ for each $K_{0}>1$. Given $K_{0}>1$, let $W$ be given by the assumption. For each $z_{0} \in \Omega_{r_{0}}^{o}$, there is a neighborhood $Z$ of $z_{0}$ and $n_{0} \geq 1$ such that $g^{n_{0}}(Z) \subset W$, and hence $g^{n}(Z) \subset W$ for all $n \geq n_{0}$. Since

$$
h_{\lambda}^{(0)}\left(g^{n}(z)\right)=G_{h_{\lambda}^{(0)}\left(c_{1}\right)} \circ \cdots \circ G_{h_{\lambda}^{(n-1)}\left(c_{1}\right)}\left(h_{\lambda}^{(n)}(z)\right),
$$

it follows that $h_{\lambda}^{(n)}$ is $K_{0}$-qc in $Z$ for each $n \geq n_{0}$. Therefore, $\check{h}_{\lambda}$ is $K_{0}$-qc in $Z$.

\section{Admissible holomorphic motions of ASymptotic inVARiance order one}

In this section, we shall prove Theorem A. Let

$$
v\left(c_{n}\right)=\left.\frac{d}{d w} G_{w}^{n-1}(w)\right|_{w=c_{1}}, n \geq 1
$$

So we have

$$
v\left(c_{n+1}\right)=L\left(c_{n}\right)+D g\left(c_{n}\right) v\left(c_{n}\right), n \geq 1,
$$

where

$$
L(z)=\left.\frac{\partial G_{w}(z)}{\partial w}\right|_{w=c_{1}} .
$$

Differentiating (2.1) at $\lambda=0$ we see from (4.1) that if $H_{\lambda}$ is a holomorphic motion of $P_{r}$ (or simply $P=\left\{c_{n}\right\} \subset P_{r}$ ) with $d H_{\lambda} /\left.d \lambda\right|_{\lambda=0}\left(c_{n}\right)=v\left(c_{n}\right), n \geq 1$, then $H_{\lambda}$ is asymptotically invariant of order 1 .

Below we will use the following formula (which follows immediately by induction):

$$
Q(z):=\left.\frac{\partial G_{w}^{q}}{\partial w}\right|_{w=c_{1}}(z)=\sum_{i=0}^{q-1} D g^{i}\left(g^{q-i}(z)\right) L\left(g^{q-i-1}(z)\right) .
$$

4.1. The hyperbolic case. The following lemma is essentially contained in 4, Lemma 6.10 and Remark 6.7], but we add the proof for the reader's convenience.

Lemma 4.1. Let $f: \mathbb{D} \rightarrow \mathbb{D}$ be a holomorphic injection such that $f(0)=0$ and $\kappa=$ $f^{\prime}(0) \in \mathbb{D} \backslash\{0\}$ and let $\Gamma: \mathbb{D} \rightarrow \mathbb{C}$ be a holomorphic function. Let $a \in \mathbb{D} \backslash\{0\}$ and $b \in \mathbb{C}$ be arbitrary. Assume that

$$
\Gamma(0) f^{\prime \prime}(0)-\Gamma^{\prime}(0)\left(f^{\prime}(0)-1\right)=0 .
$$

Then there exists a holomorphic map $w: \mathbb{D} \rightarrow \mathbb{C}$ such that $w(a)=b$ and

$$
w \circ f(z)=\Gamma(z)+f^{\prime}(z) w(z) .
$$

Proof. Let $\varphi: \mathbb{D} \rightarrow \mathbb{C}$ denote the Koenigs linearization, i.e., $\varphi$ is a conformal map onto its image, with $\varphi(0)=0, \varphi^{\prime}(0)=1$ and $\varphi(f(z))=\kappa \varphi(z)$ for all $z \in \mathbb{D}$. Write $\widetilde{w}(z)=$ $w \circ \varphi^{-1}(z) \varphi^{\prime}\left(\varphi^{-1}(z)\right)$, and $\widetilde{\Gamma}(z)=\Gamma\left(\varphi^{-1}(z)\right) \varphi^{\prime}\left(f \circ \varphi^{-1}(z)\right)$. Since $\varphi^{\prime}(f(z)) f^{\prime}(z)=\kappa \varphi^{\prime}(z)$, the equation 4.5 is reduced to the following form:

$$
\widetilde{w}(\kappa z)=\widetilde{\Gamma}(z)+\kappa \widetilde{w}(z) .
$$

From $\varphi(f(z))=\kappa \varphi(z)$, we obtain

$$
\varphi^{\prime}(f(z)) f^{\prime}(z)=\kappa \varphi^{\prime}(z)
$$


and

$$
\varphi^{\prime \prime}(f(z)) f^{\prime}(z)^{2}+\varphi^{\prime}(f(z)) f^{\prime \prime}(z)=\kappa \varphi^{\prime \prime}(z),
$$

hence $\varphi^{\prime \prime}(0)=f^{\prime \prime}(0) /\left(\kappa-\kappa^{2}\right)$. Thus the condition 4.4 is equivalent to $\widetilde{\Gamma}^{\prime}(0)=0$. Under this condition,

$$
u(z)=-\kappa^{-1} \sum_{n=0}^{\infty} \widetilde{\Gamma}^{\prime}\left(\kappa^{n} z\right)
$$

defines a holomorphic map satisfying $\kappa u(\kappa z)=\widetilde{\Gamma}^{\prime}(z)+\kappa u(z)$. Let $\widetilde{w}$ be a holomorphic map such that $\tilde{w}(\varphi(a))=\varphi^{\prime}(a) b, \tilde{w}(0)=\tilde{\Gamma}(0) /(1-\kappa)$ and such that $\tilde{w}^{\prime \prime}(z)=u^{\prime}(z)$. Then it solves the equation (4.6) and $w(z)=\widetilde{w} \circ \varphi(z) / \varphi^{\prime}(z)$ solves the equation 4.5 with $w(a)=b$.

Proof of Theorem A in the attracting case. Let $\delta>0$ be such that $g^{q}$ maps $B\left(a_{0}, \delta\right)$ injectively into $B\left(a_{0}, \delta\right)$ and let $N$ be such that $c_{N} \in B\left(a_{0}, \delta\right)$. By assumption,

$$
Q\left(a_{0}\right) D^{2} g^{q}\left(a_{0}\right)=Q^{\prime}\left(a_{0}\right)(\kappa-1) .
$$

So by Lemma 4.1, there is a holomorphic map $w: B\left(a_{0}, \delta\right) \rightarrow \mathbb{C}$ such that

$$
w\left(g^{q}(z)\right)=Q(z)+D g^{q}(z) w(z) \text { for } z \in B\left(a_{0}, \delta\right),
$$

and $w\left(c_{N}\right)=v\left(c_{N}\right)$. The function $w$ extends naturally to a map $w: P_{r_{0}} \rightarrow \mathbb{C}$ that satisfies $w(g(z))=L(z)+D g(z) w(z)$ which is holomorphic in $\Omega_{r_{0}}^{o}$. Since $w\left(c_{N}\right)=v\left(c_{N}\right)$, and $v\left(c_{n+1}\right)=L\left(c_{n}\right)+D g\left(c_{n}\right) v\left(c_{n}\right)$, it follows that $w\left(c_{n}\right)=v\left(c_{n}\right)$ for all $n \geq N$.

In particular, $w \mid P_{\delta / 2}$ is Lipschitz. Thus $H_{\lambda}(z):=z+\lambda w(z)$ defines a holomorphic motion of $P_{\delta / 2}$ over $\left(\mathbb{D}_{\varepsilon}, 0\right)$ for some $\varepsilon>0$. Since every point in $\Omega_{r_{0}}$ eventually lands in $\bigcup B\left(a_{j}, \delta / 2\right)$, applying Lemma 3.2 completes the proof.

\subsection{The parabolic case.}

Lemma 4.2. Let $W$ be a neighborhood of 0 in $\mathbb{C}$ and let $f, \Gamma: W \rightarrow \mathbb{C}$ be holomorphic functions with $f(0)=0, f^{\prime}(0)=e^{2 \pi i l / p}$ and $D^{p+1} f^{p}(0) \neq 0$, where $l, p \in \mathbb{Z}, p \geq 1$ and $(l, p)=1$, and with

$$
\Gamma^{\prime}(0)\left(f^{\prime}(0)-1\right)=\Gamma(0) f^{\prime \prime}(0) .
$$

Let $P=\left\{z_{n}: n \geq 1\right\} \subset W$ be an infinite orbit of $f$ such that $z_{n}=f^{n-1}\left(z_{1}\right) \rightarrow 0$ and let $v: P \rightarrow \mathbb{C}$ be a function such that

$$
v(z) f^{\prime}(z)+\Gamma(z)=v(f(z)), \text { for each } z \in P .
$$

Then $v$ extends to a $C^{1}$ map $V: \mathbb{C} \rightarrow \mathbb{C}$ such that $\bar{\partial} V(0)=0$.

Proof. Step 1. Let us prove that there exists a polynomial $h$ and a holomorphic function $\widehat{\Gamma}$ defined near 0 such that

$$
D^{j} \widehat{\Gamma}(0)=0, \text { for } j=0,1, \cdots, p+1,
$$

and such that

$$
\mathbf{v}\left(z_{n}\right)\left(f^{p}\right)^{\prime}\left(z_{n}\right)+\widehat{\Gamma}\left(z_{n}\right)=\mathbf{v}\left(z_{n+p}\right)
$$

for all $n$ large enough, where $\mathbf{v}(z)=v(z)+h(z)$.

We first deal with the case $p=1$. Then $f^{\prime \prime}(0) \neq 0$ and $\Gamma(0)=0$. Define $h_{1}(z)=$ $\Gamma^{\prime}(0) / f^{\prime \prime}(0), \Gamma_{1}=\Gamma(z)-h_{1}(z) f^{\prime}(z)+h_{1}(f(z))$ and $v_{1}(z)=v(z)+h_{1}(z)$. Then $\Gamma_{1}(0)=$ $\Gamma_{1}^{\prime}(0)=0$ and $v_{1}(z) f^{\prime}(z)+\Gamma_{1}(z)=v_{1}(f(z))$ for each $z \in P$. Let now $b_{1}=\Gamma_{1}^{\prime \prime}(0) / f^{\prime \prime}(0)$, $h(z)=h_{1}(z)+b_{1} z$. Then $\widehat{\Gamma}(z)=\Gamma_{1}(z)-b_{1}\left(z f^{\prime}(z)-f(z)\right)$ and $\mathbf{v}(z)=v_{1}(z)+b_{1} z=$ $v(z)+h(z)$ satisfy the desired property.

Now assume $p>1$. Claim. For any $1 \leq k \leq p$, there is a polynomial $h_{1, k}$ such that $\Gamma_{1, k}(z)=\Gamma(z)-h_{1, k}(z) f^{\prime}(z)+h_{1, k}(f(z))$ satisfies

$$
\Gamma_{1, k}(z)=O\left(z^{k+1}\right) .
$$


Let us prove this by induction. For $k=1$, define $h_{1,1}(z)=\Gamma(0) /\left(f^{\prime}(0)-1\right)$. Then the claim follows from (4.7). Assume now the claim holds for some $1 \leq k<p$. Let $A$ be such that $\Gamma_{1, k}(z)=A z^{k+1}+O\left(z^{k+2}\right)$. Define $h_{1, k+1}(z)=h_{1, k}(z)+b z^{k+1}$, where $b=A /\left(f^{\prime}(0)-f^{\prime}(0)^{k+1}\right)$. Then

$$
\begin{aligned}
\Gamma_{1, k+1}(z) & =\Gamma_{1, k}(z)-b z^{k+1} f^{\prime}(z)+b f(z)^{k+1} \\
& =A z^{k+1}-b f^{\prime}(0) z^{k+1}+b f^{\prime}(0)^{k+1} z^{k+1}+O\left(z^{k+2}\right)=O\left(z^{k+2}\right)
\end{aligned}
$$

completing the proof of the claim.

Define $h_{1}=h_{1, p}, \Gamma_{1}(z)=\Gamma_{1, p}, v_{1}(z)=v(z)+h_{1}(z)$ and

$$
\widetilde{\Gamma}_{1}(z)=D f^{p}(z) \sum_{k=1}^{p} \frac{\Gamma_{1}\left(f^{k-1}(z)\right)}{D f^{k}(z)}=O\left(z^{p+1}\right) .
$$

Then $v_{1}(z) f^{\prime}(z)+\Gamma_{1}(z)=v_{1}(f(z))$ for each $z \in P$, hence

$$
v_{1}(z)\left(f^{p}\right)^{\prime}(z)+\widetilde{\Gamma}_{1}(z)=v_{1}\left(f^{p}(z)\right), z \in P .
$$

Now take $b_{1}:=\widetilde{\Gamma}_{1}^{(p+1)}(0) /\left(p D^{p+1} f^{p}(0)\right), h(z)=h_{1}(z)+b_{1} z, \mathbf{v}(z)=v_{1}(z)+b_{1} z$ and $\widehat{\Gamma}=\widetilde{\Gamma}_{1}(z)-b_{1}\left(z\left(f^{p}\right)^{\prime}(z)-f^{p}(z)\right)$. Then $\widehat{\Gamma}$ and $\mathbf{v}$ satisfy 4.8$)$ and 4.9$)$.

Step 2. Let us prove that there exist $M>0$ and $\sigma>0$ such that

$$
\left|\mathbf{v}\left(z_{n}\right)-\mathbf{v}\left(z_{n+p}\right)\right| \leq M\left|z_{n}-z_{n+p}\right|^{1+\sigma} .
$$

Let $A=D^{p+1} f^{p}(0) /(p+1) ! \neq 0$. By the Leau-Fatou Flower Theorem, see Appendix B, $\left|\left(f^{p}\right)^{\prime}\left(z_{n}\right)\right| \sim 1-|A|(p+1)\left|z_{n}\right|^{p}$ and

$$
\frac{z_{n}}{z_{n+p}} \sim \frac{1}{1-|A|\left|z_{n}\right|^{p}}
$$

Fix an arbitrary $\varepsilon \in(0,1)$ and $\delta \in(0,|A|(p-\varepsilon))$. There exists $n_{0}$ such that

$$
\gamma_{n}:=\left(\frac{\left|z_{n}\right|}{\left|z_{n+p}\right|}\right)^{1+\varepsilon}\left|\left(f^{p}\right)^{\prime}\left(z_{n}\right)\right| \leq 1-\delta\left|z_{n}\right|^{p}
$$

holds for all $n \geq n_{0}$. Write $w_{n}=\left|\mathbf{v}\left(z_{n}\right)\right| /\left|z_{n}\right|^{1+\varepsilon}$ and let $C_{0}$ be a constant such that $\left|\widehat{\Gamma}\left(z_{n}\right)\right| \leq C_{0}\left|z_{n+p}\right|^{p+2}$ for all $n$ which exists by 4.8 , 4.11. Then for all $n \geq n_{0}$,

$$
w_{n+p} \leq \gamma_{n} w_{n}+\frac{\left|\widehat{\Gamma}\left(z_{n}\right)\right|}{\left|z_{n+p}\right|^{1+\varepsilon}} \leq\left(1-\delta\left|z_{n}\right|^{p}\right) w_{n}+C_{0}\left|z_{n+p}\right|^{p+1-\varepsilon} .
$$

Let $M_{0}>0$ be such that $w_{n} \leq M_{0}$ for all $n \leq n_{0}$ and such that $C_{0}\left|z_{n+p}\right|^{1-\varepsilon}<\delta M_{0}$ for all $n \geq n_{0}$. Then by induction using that $\left|z_{n+p}\right|<\left|z_{n}\right|$, we obtain $w_{n} \leq M_{0}$ for all $n$.

Finally

$$
\begin{aligned}
\mathbf{v}\left(z_{n+p}\right)-\mathbf{v}\left(z_{n}\right) & =\widehat{\Gamma}\left(z_{n}\right)+\left(\left(f^{p}\right)^{\prime}\left(z_{n}\right)-1\right) \mathbf{v}\left(z_{n}\right)=O\left(z_{n}^{p+2}\right)+O\left(z_{n}^{p+1+\varepsilon}\right) \\
= & O\left(z_{n}^{p+1+\varepsilon}\right)=O\left(\left|z_{n}-z_{n+p}\right|^{(p+1+\varepsilon) /(p+1)}\right)=O\left(\left|z_{n}-z_{n+p}\right|^{1+\varepsilon /(p+1)}\right) .
\end{aligned}
$$

Now, let us prove that $\mathbf{v}: P \rightarrow \mathbb{C}$ extends continuous to 0 by showing that

$$
\lim _{n \rightarrow \infty} \mathbf{v}\left(z_{n}\right)=0 \text {. }
$$

It follows from 4.10 that given $n$ there exists $\lim _{j \rightarrow \infty} \mathbf{v}\left(z_{n+j p}\right)$ so it is enough to prove that the latter limit is 0 for any $n$. Assuming by a contradiction that this is not the case for some $n_{0}$ and using (4.8) and (4.9) we get (replacing if necessary $n_{0}$ by $n_{0}+j_{0} p$ with a big $j_{0}$ ),

$$
\left|\mathbf{v}\left(z_{n_{0}+j p}\right)\right| \leq\left|\mathbf{v}\left(z_{n_{0}}\right)\right| \Pi_{i=1}^{j-1}\left|\left(f^{p}\right)^{\prime}\left(z_{n_{0}+i p}\right)\right|\left(1+O\left(\left|z_{n_{0}+i p}\right|^{p+2}\right)\right) \rightarrow 0
$$

as $j \rightarrow \infty$ because $\Pi_{i=1}^{j-1}\left(f^{p}\right)^{\prime}\left(z_{n_{0}+i p}\right)=\left(f^{j p}\right)^{\prime}\left(z_{n_{0}}\right) \rightarrow 0$ and because $\sum_{i=0}^{\infty}\left|z_{n_{0}+i p}\right|^{p+2} \leq$ $\sum_{i=0}^{\infty} O\left(i^{-(p+2) / p}\right)<\infty$, a contradiction.

Step 3. We shall now prove that $\mathbf{v}: P \rightarrow \mathbb{C}$ extends to a $C^{1}$ function $\mathbf{V}: \mathbb{C} \rightarrow \mathbb{C}$ such that $\partial \mathbf{V}(0)=\bar{\partial} \mathbf{V}(0)=0$. Once this is proved, we obtain a desired extension $V$ of $v$ by setting $V=\mathbf{V}-h$. 
Indeed, by the Leau-Fatou Flower Theorem (see Appendix B) for each $j=0,1, \ldots, p-$ 1 , there exists $\theta_{j} \in \mathbb{R}$ with $A e^{2 \pi i \theta_{j}}=-|A|<0, \theta_{j+1}=\theta_{j}+2 \pi / p$ such that the argument $z_{n p+j}$ converges to $\theta_{j}$ as $n \rightarrow \infty$. Moreover, the argument of $z_{n+p}-z_{n}=A z_{n}^{p}\left(1+o\left(z_{n}\right)\right)$ converges to $\pi$. Therefore, there is a $C^{1}$ diffeomorphism $H: \mathbb{C} \rightarrow \mathbb{C}$ with $H(z)=z+o(|z|)$ near $z=0$ such that $H^{-1}\left(z_{n p+j}\right)$ lies in order on the ray $\theta=\theta_{j}$. Write $z_{n}^{\prime}=H^{-1}\left(z_{n}\right)$. Then $\mathbf{v} \circ H\left(z_{n}^{\prime}\right)-\mathbf{v} \circ H\left(z_{n+p}^{\prime}\right)=o\left(\left|z_{n}^{\prime}-z_{n+p}^{\prime}\right|\right)$, $\mathbf{v} \circ H \mid H^{-1}(P)$, and hence $\mathbf{v}$, extends to a $C^{1}$ map defined on $\mathbb{C}$ with zero partial derivatives at 0 .

Proof of Theorem A in the parabolic case. Define $v\left(c_{n}\right)=\left.\frac{d G_{w}^{n-1}(w)}{d w}\right|_{w=c_{1}}$. By Proposition 4.2 applying for $f(z)=g^{q}\left(z+a_{j}\right)$ and $\Gamma(z)=Q\left(z+a_{j}\right)$ for each $j$, the vector field $v$ on $P$ extends to a $C^{1}$ vector field $V$ in $\mathbb{C}$ such that $\bar{\partial} V(z) \rightarrow 0$ as $z \rightarrow a_{j}$, $j=0,1, \ldots, q-1$. We can surely make the extension compactly supported. So $\mu=\bar{\partial} V$ is a qc vector field. By the Measurable Riemann Mapping Theorem, there is a holomorphic motion $h_{\lambda}$ of $\mathbb{C}$ over some disk $\mathbb{D}_{\varepsilon}$, such that $\bar{\partial} h_{\lambda}=\lambda \mu \partial h_{\lambda}$ and $h_{\lambda}(z)=z+o(1)$ as $z \rightarrow \infty$. In particular, $h_{\lambda}$ defines a holomorphic motion of $P_{r_{0}}$ which is asymptotically invariant of order 1 and

$$
\left.\frac{d}{d \lambda} h_{\lambda}(z)\right|_{\lambda=0}=V(z), \forall z \in P
$$

To complete the proof, we shall apply Lemma 3.2 Let us verify the conditions. For each $K>1$, choose $r$ small enough so that $|\bar{\partial} V|<(K-1)(K+1)$ holds in $\Omega_{r} \subset \bigcup_{j} \overline{B\left(a_{j}, r\right)}$ and let $W=\Omega_{r}$. Both conditions are clearly satisfied.

\section{Averaging And PROMoting asymptotic inVARiance}

In this section, we prove Theorem B.

5.1. The averaging process. Suppose that $(g, G)_{W}$ is a local holomorphic deformation of a marked map $g: U \rightarrow \mathbb{C}$ which has the lifting property of some set $K$ with $P \subset K$ and $g(K) \subset K$. Let $h_{\lambda}$ be a holomorphic motion of $K$ over $\left(\mathbb{D}_{\epsilon}, 0\right)$. By the lifting property, there is $\varepsilon^{\prime}>0$ and a sequence $h_{\lambda}^{(k)}, k=0,1, \cdots$ of holomorphic motions of $K$ over $\left(\mathbb{D}_{\epsilon^{\prime}}, 0\right)$ so that $h_{\lambda}^{(0)}=h_{\lambda}$ and $h_{\lambda}^{(k+1)}$ is the lift of $h_{\lambda}^{(k)}$, for each $k \geq 0$.

Let $H_{\lambda}$ be a (locally uniform) limit for some subsequence $k_{i} \rightarrow \infty$ of

$$
\tilde{h}_{\lambda}^{(k)}:=\frac{1}{k} \sum_{i=0}^{k-1} h_{\lambda}^{(i)} .
$$

The following proposition is proved in [24, Lemma 2.12], [25, Lemma 6.4].

Proposition 5.1. Assume $h_{\lambda}^{0}$ is asymptotically invariant of some order $m$, i.e.

$$
h_{\lambda}^{(k+1)}\left(c_{j}\right)-h_{\lambda}^{(k)}\left(c_{j}\right)=O\left(\lambda^{m+1}\right), \quad j=1,2, \cdots \text { as } \lambda \rightarrow 0
$$

for $k=0$ (hence, for all $k$ ). Then $H_{\lambda}$ is asymptotically invariant of order $m+1$, i.e.

$$
\hat{H}_{\lambda}\left(c_{j}\right)-H_{\lambda}\left(c_{j}\right)=O\left(\lambda^{m+2}\right), \quad j=1,2, \cdots \text { as } \lambda \rightarrow 0 .
$$

When $K$ is finite, then $H_{\lambda}$, when restricting $\lambda$ to a smaller disk, is automatically a holomorphic motion. However, this is not necessarily the case when $K$ has infinite cardinality. We solve this issue by considering holomorphic motions which are 'almost' conformal near $\mathcal{O}$, using distortion estimates.

\subsection{The attracting case.}

Proof of Theorem B in the attracting case. Let $h_{\lambda}$ be an admissible holomorphic motion of $P_{r_{0}}$ over $\left(\mathbb{D}_{\varepsilon}, 0\right)$ which is asymptotically invariant of order $m$ and let $h_{\lambda}^{(k)}, \hat{h}_{\lambda}^{(k)}$ and $H_{\lambda}$ be as in Subsection 5.1 .

Let us prove that there is $r \in\left(0, r_{0}\right)$ and $\varepsilon_{1}>0$ such that $H_{\lambda}$ is an admissible holomorphic motion of $P_{r}$ over $\left(\mathbb{D}_{\varepsilon_{1}}, 0\right)$. Indeed, by definition of the lifting property, 
there exists $M>0$ and $\varepsilon_{2}>0$ such that $\left|h_{\lambda}^{(k)}(z)\right| \leq M$ for all $z \in P_{r_{0}}$ and $\lambda \in \mathbb{D}_{\varepsilon_{2}}$. By Slodowski's theorem, there exists $M^{\prime}>0$ such that $h_{\lambda}^{(k)}$ extends to a holomorphic motion of $\mathbb{C}$ over $\mathbb{D}_{\varepsilon_{2}}$ such that $h_{\lambda}^{(k)}(z)=z$ whenever $|z|>M^{\prime}$. By Bers-Royden's Theorem [5], there exists $K(\lambda)>1$ for each $\lambda \in \mathbb{D}_{\varepsilon_{2}}$ with $K(\lambda) \rightarrow 1$ as $\lambda \rightarrow 0$ such that for each $k=0,1, \ldots, h_{\lambda}^{(k)}$ is $K(\lambda)$-qc. Thus for each $\delta>0$ there exists $\varepsilon(\delta)>0$ such that $\left|h_{\lambda}^{(k)}(z)-z\right| \leq \delta$ for all $z \in P_{r_{0}}$ and $\lambda \in \mathbb{D}_{\varepsilon(\delta)}$. Since $a_{j}$ is in the interior of $P_{r_{0}}$, it follows that there is $\varepsilon_{3}>0$ such that $\left|\left(h_{\lambda}^{(k)}\right)^{\prime}\left(a_{j}\right)-1\right|<\frac{1}{3}$ for all $\lambda \in \mathbb{D}_{\varepsilon_{3}}$. By the Koebe Distortion Theorem, there exists $r \in\left(0, r_{0}\right)$ such that for any $z_{1}, z_{2} \in B\left(a_{j}, r\right), z_{1} \neq z_{2}$,

$$
\left|\frac{h_{\lambda}^{(k)}\left(z_{1}\right)-h_{\lambda}^{(k)}\left(z_{2}\right)}{z_{1}-z_{2}}-1\right|<\frac{1}{2},
$$

which implies that

$$
\left|\frac{H_{\lambda}\left(z_{1}\right)-H_{\lambda}\left(z_{2}\right)}{z_{1}-z_{2}}-1\right| \leq \frac{1}{2},
$$

hence $H_{\lambda}$ is a holomorphic motion of $\Omega_{r}$ over $\mathbb{D}_{\varepsilon_{3}}$. As $P \backslash \Omega_{r}$ is finite, the statement follows by choosing $\varepsilon_{1}$ sufficiently small.

To complete the proof, we extend $H_{\lambda}$ to a holomorphic motion of $P_{r_{0}}$ in an arbitrary way and then apply Lemma 3.2 as follows: simply take $W=\Omega_{r}$ for each $K>1$.

5.3. The parabolic case. The parabolic case is more complicated. We shall need the following distortion lemma:

Lemma 5.2. Given a positive integer $p, \alpha \in(0,1), M>0$ and $R>0$ with $3 R<M$ and $(3 R)^{\alpha}<\pi /(4 p)$, there is $K_{0}>1$ such that if $H: B(0, M) \rightarrow B(0, M)$ is a $K_{0}-q c$ map satisfying $H \mid \partial B(0, M)=i d$ and

$$
\bar{\partial} H=0 \text { a.e. on } B(0,3 R) \backslash \mathcal{C}(R),
$$

where

$$
\mathcal{C}(R)=\left\{r e^{2 \pi i t}: 0<r<3 R,|t-(2 k+1) \pi / p|<r^{\alpha} \text { for some } k=0,1, \ldots, p-1\right\},
$$

then for any $z_{1}, z_{2} \in \mathcal{C}^{\prime}(R)=\left\{r e^{2 \pi i t}: 0<r<R,|t-2 k \pi / p|<r^{\alpha}\right.$ for some $k=$ $0,1, \ldots, p-1\}$, we have

$$
\left|H\left(z_{1}\right)-H\left(z_{2}\right)-\left(z_{1}-z_{2}\right)\right| \leq \frac{1}{2}\left|z_{1}-z_{2}\right| .
$$

Proof. Choose $q^{\prime} \in(1,1+\alpha / 2)$ and let $p^{\prime}>1$ be such that $1 / p^{\prime}+1 / q^{\prime}=1$. Let $D=B(0,3 R)$. Let $\varepsilon>0$ be a small constant to be determined. Since $H \mid \partial B(0, M)=i d$, it is well-known, see for example [1, Chapter V], that provided that $K_{0}$ is sufficiently close to 1 ,

$$
\iint_{D}|\bar{\partial} H|^{p^{\prime}} d|u|^{2}<\varepsilon^{p^{\prime}}, \text { and }|H(z)-z|<\varepsilon R, \text { for all } z \in D .
$$

Since $H$ is ACL, we can apply the Cauchy-Pompeiu Formula

$$
H(z)-z=\frac{1}{2 \pi i} \int_{\partial D} \frac{H(u)-u}{u-z} d u-\frac{1}{\pi} \iint_{D} \frac{\bar{\partial} H(u)}{u-z}|d u|^{2},
$$

for $z \in D$. For $z_{1}, z_{2} \in \mathcal{C}^{\prime}(R)$, and $u \in \partial D$, we have $\left|u-z_{1}\right| \geq 2 R,\left|u-z_{2}\right| \geq 2 R$, so

$$
\begin{gathered}
\left|\frac{1}{2 \pi i} \int_{\partial D} \frac{H(u)-u}{u-z_{1}} d u-\frac{1}{2 \pi i} \int_{\partial D} \frac{H(u)-u}{u-z_{2}} d u\right|=\left|\left(z_{1}-z_{2}\right) \frac{1}{2 \pi i} \int_{\partial D} \frac{H(u)-u}{\left(u-z_{1}\right)\left(u-z_{2}\right)} d u\right| \\
\leq\left|z_{1}-z_{2}\right| \frac{1}{2 \pi} \frac{\varepsilon R}{4 R^{2}} \cdot 2 \pi \cdot 3 R<\frac{1}{4}\left|z_{1}-z_{2}\right|,
\end{gathered}
$$


where we choose $\varepsilon$ small enough to obtain the last inequality. For $u \in \mathcal{C}(R)$, we have $\left|u-z_{j}\right| \geq \rho|u|$, where $\rho=\rho(p, \alpha, R)>0$ is a constant. Thus

$$
\begin{aligned}
\iint_{\mathcal{C}(R)} \frac{1}{\left|u-z_{1}\right|^{q^{\prime}}\left|u-z_{2}\right|^{q^{\prime}}} d|u|^{2} \leq \frac{1}{\rho^{2 q^{\prime}}} \sum_{k=0}^{p-1} \int_{0}^{3 R} \int_{|t-(2 k+1) \pi / p|<r^{\alpha}} \frac{1}{r^{2 q^{\prime}}} r d t d r \\
=\frac{2 p}{\rho^{2 q^{\prime}}} \frac{(3 R)^{2+\alpha-2 q^{\prime}}}{2+\alpha-2 q^{\prime}}=: C .
\end{aligned}
$$

Therefore,

$$
\begin{gathered}
\left.\left|\iint_{D} \frac{\bar{\partial} H(u)}{u-z_{1}} d\right| u\right|^{2}-\iint_{D} \frac{\bar{\partial} H(u)}{u-z_{2}} d|u|^{2}|=| \iint_{\mathcal{C}(R)} \frac{\bar{\partial} H(u)}{u-z_{1}} d|u|^{2}-\iint_{\mathcal{C}(R)} \frac{\bar{\partial} H(u)}{u-z_{2}} d|u|^{2} \mid \\
=\left.\left|z_{1}-z_{2}\right|\left|\iint_{\mathcal{C}(R)} \frac{\bar{\partial} H(u)}{\left(u-z_{1}\right)\left(u-z_{2}\right)} d\right| u\right|^{2} \mid \\
\leq\left|z_{1}-z_{2}\right|\left(\iint_{\mathcal{C}(R)}|\bar{\partial} H(u)|^{p^{\prime}} d|u|^{2}\right)^{1 / p^{\prime}}\left(\iint_{\mathcal{C}(R)} \frac{1}{\left|u-z_{1}\right|^{\prime}\left|u-z_{2}\right|^{\prime}} d|u|^{2}\right)^{1 / q^{\prime}} \\
\leq\left|z_{1}-z_{2}\right| \varepsilon C^{1 / q^{\prime}}<\pi\left|z_{1}-z_{2}\right| / 4,
\end{gathered}
$$

where, once again, we choose $\varepsilon>0$ small enough to obtain the last inequality. The lemma follows.

Proof of Theorem B in the parabolic case. Extend each $h_{\lambda}^{(k)}$ to a holomorphic motion of $\mathbb{C}$ over $\left(\mathbb{D}_{\varepsilon^{\prime}}, 0\right)$ and such that $h_{\lambda}^{(k)}(z)=z$ for all $\lambda \in \mathbb{D}_{\varepsilon^{\prime}}, k$ and $|z|>M^{\prime}$. Fix $\alpha \in(0,1)$. Let $R=\tau\left(r_{0}\right) / 3$ be given by Lemma B.1 (1) and let $\tilde{K}=K_{0}\left(p, \alpha, M^{\prime}, R\right)$ be given by Lemma 5.2 By [5], there exists $\varepsilon_{1}>0$ such that $h_{\lambda}^{(k)}$ is $\tilde{K}$-qc for all $\lambda \in \mathbb{D}_{\varepsilon_{1}}$. Thus for each $z_{1}, z_{2} \in \mathcal{C}_{j}^{\prime}, 0 \leq j<p, z_{1} \neq z_{2}$, and any $k \geq 0, \lambda \in \mathbb{D}_{\varepsilon_{1}}$,

$$
\left|\frac{h_{\lambda}^{(k)}\left(z_{1}\right)-h_{\lambda}^{(k)}\left(z_{2}\right)}{z_{1}-z_{2}}-1\right| \leq \frac{1}{2},
$$

hence

$$
\left|\frac{H_{\lambda}\left(z_{1}\right)-H_{\lambda}\left(z_{2}\right)}{z_{1}-z_{2}}-1\right| \leq \frac{1}{2} .
$$

It follows that $H_{\lambda}$ is a holomorphic motion of $P \cup \bigcup_{j} \mathcal{C}_{j}^{\prime}(\tau)$ over $\mathbb{D}_{\varepsilon_{2}}$ of asymptotic invariance of order $m+1$. By extending it to a holomorphic motion of $P_{r_{0}}$ in an arbitrary way and applying Lemma 3.2 by taking $W=\bigcup \mathcal{C}_{j}^{\prime}(\tau)$ for all $K_{0}>1$, we complete the proof.

\section{ASYMPtotic INVARIANCE OF AN ARBITRARILY LARGE ORDER}

In this section, we shall prove Theorem C. For each $m \geq 1$, let $h_{\lambda, m}$ be a holomorphic motion of $\bar{P}$ over $\left(\mathbb{D}_{\varepsilon_{m}}, 0\right)$ for some $\varepsilon_{m}>0$ which is asymptotically invariant of order $m$ such that $h_{\lambda, m}\left(c_{1}\right)=c_{1}+\lambda+O\left(\lambda^{2}\right)$ as $\lambda \rightarrow 0$. Claim: we may assume that the holomorphic motion $h_{\lambda, m}$ satisfies $h_{\lambda, m}\left(c_{1}\right)=c_{1}+\lambda$. Indeed, for each $m$ take a reparametrisation $\lambda=\lambda_{m}(\mu)$ so that for $\hat{h}_{\mu, m}:=h_{\lambda_{m}(\mu), m}$ we have $\hat{h}_{\mu, m}\left(c_{1}\right)=c_{1}+$ $\mu$. Then $\hat{h}_{\mu, m}$ is still asymptotically invariant of order $m$. That is, $G_{\hat{h}_{\mu}\left(c_{1}\right)}\left(\hat{h}_{\mu}(z)\right)=$ $\hat{h}_{\mu}(g(z))+O\left(\mu^{m+1}\right)$. Renaming the new holomorphic motion again $h_{\lambda, m}$ the claim follows.

Then for each $k \geq 1$,

$$
h_{\lambda, m}\left(c_{k}\right)=G_{\lambda+c_{1}}^{k-1}\left(\lambda+c_{1}\right)+O\left(\lambda^{m+1}\right)
$$

and so the first $m$ terms in the Taylor series of $h_{\lambda}\left(c_{k}\right)$ is fixed according to this formula and therefore $h_{\lambda, m}\left(c_{k}\right)-h_{\lambda, m+1}\left(c_{k}\right)=O\left(\lambda^{m+1}\right)$. Assume without loss of generality that 
$c_{n q+1} \rightarrow a_{1}$ as $n \rightarrow \infty$ and define $\varphi_{m}(\lambda):=\lim _{n \rightarrow \infty} h_{\lambda, m}\left(c_{n q+1}\right)-a_{1}:=h_{\lambda, m}\left(a_{1}\right)-a_{1}$. Then

$$
G_{c_{1}+\lambda}^{q}\left(\varphi_{m}(\lambda)+a_{1}\right)=\varphi_{m}(\lambda)+a_{1}+O\left(\lambda^{m+1}\right) .
$$

Lemma 6.1. There is a function $\varphi(\lambda)$, holomorphic near $\lambda=0$ and $m_{0}$, such that

$$
G_{c_{1}+\lambda}^{q}\left(\varphi(\lambda)+a_{1}\right)=\varphi(\lambda)+a_{1}
$$

and such that for each $m \geq m_{0}$,

$$
\varphi_{m}(\lambda)-\varphi(\lambda)=O\left(|\lambda|^{m / 3}\right) \text { as } \lambda \rightarrow 0 .
$$

Proof. In the case $\kappa=D g^{q}\left(a_{1}\right) \neq 1$, we simply take $\varphi(\lambda)$ so that $\varphi(\lambda)+a_{1}$ is the fixed point of $G_{c_{1}+\lambda}^{q}$ obtained as analytic continuation of $a_{1}$. It is easy to check that 6.2 holds for all $m \geq 1$ with an even better error term: $O\left(\lambda^{m+1}\right)$ instead of $O\left(|\lambda|^{m / 3}\right)$.

Now assume $\kappa=1$ and consider the map $\Phi(\lambda, z)=G_{c_{1}+\lambda}^{q}\left(z+a_{1}\right)-z-a_{1}$ which is holomorphic in a neighborhood of the origin in $\mathbb{C}^{2}$. Clearly, $\Phi(0, z)$ is not identically zero, so by Weierstrass' Preparation Theorem, there is a Weierstrass polynomial

$$
Q(\lambda, z)=z^{2}+2 u(\lambda) z+v(\lambda)=(z+u(\lambda))^{2}+v(\lambda)-u(\lambda)^{2}
$$

such that $\Phi(\lambda, z)=Q(\lambda, z) R(\lambda, z)$, where $R$ is a holomorphic function near the origin and $R(0,0) \neq 0$, and $u, v$ are holomorphic near the origin in $\mathbb{C}$ with $u(0)=v(0)=0$. Consider the discriminant $\Delta(\lambda)=u(\lambda)^{2}-v(\lambda)$ which satisfies $\Delta(0)=0$. By 6.1 , for each $m$,

$$
\left(\varphi_{m}(\lambda)+u(\lambda)\right)^{2}-\Delta(\lambda)=O\left(\lambda^{m+1}\right) .
$$

Let us distinguish two cases.

Case 1. $\Delta(\lambda) \equiv 0$ (for $\lambda \in \mathbb{D}_{\varepsilon}$ for some $\varepsilon>0$ ). Then $-u(\lambda)$ is the only zero of $Q(\lambda, z)$ near 0. By 6.3), $\varphi_{m}(\lambda)+u(\lambda)=O\left(|\lambda|^{(m+1) / 2}\right)$. So the claim holds with $\varphi=-u$.

Case 2. $\Delta(\lambda) \not \equiv 0$. Then there is $n_{0} \geq 1$ and $A \neq 0$ such that $\Delta(\lambda)=A \lambda^{n_{0}}+O\left(\lambda^{n_{0}+1}\right)$. Assume $m \geq n_{0}$. By (6.3),

$$
\left(\varphi_{m}(\lambda)+u(\lambda)\right)^{2}=A \lambda^{n_{0}}+O\left(\lambda^{n_{0}+1}\right)
$$

which implies that $n_{0}$ is even. Therefore, there exists holomorphic functions $\varphi_{ \pm}(\lambda)$ such that $Q(\lambda, z)=\left(z-\varphi_{+}(\lambda)\right)\left(z-\varphi_{-}(\lambda)\right)$. The lemma holds for either $\varphi(\lambda)=\varphi_{-}(\lambda)$ or $\varphi(\lambda)=\varphi_{-}(\lambda)$.

Completion of the proof of Theorem $C$. We want to show that $\sigma(\lambda):=D G_{c_{1}+\lambda}^{q}(\varphi(\lambda)+$ $\left.a_{1}\right)$ is constant. Arguing by contradiction, assume that this is not the case. Then there exists $m_{1} \geq 1$ and $A \neq 0$, such that

$$
\sigma(\lambda)-\sigma(0)=3 A \lambda^{m_{1}}+O\left(\lambda^{m_{1}+1}\right) .
$$

Fix $m \geq \max \left(3\left(m_{1}+1\right), m_{0}\right)$. There exists $\varepsilon^{\prime}=\varepsilon_{m}^{\prime}>0$ such that the following hold:

- There exist admissible holomorphic motions $h_{\lambda, m}^{k}$ of $\bar{P}_{r_{0}}$ over $\mathbb{D}_{2 \varepsilon^{\prime}}, k=0,1, \ldots$, such that $h_{\lambda, m}^{0}=h_{\lambda, m}, h_{\lambda, m}\left(c_{1}\right)=c_{1}+\lambda$ and such that $h_{\lambda, m}^{k+1}$ is the lift of $h_{\lambda, m}^{k}$ for each $k$, and the sequence $\left\{h_{\lambda, m}^{k}(x)\right\}$ is uniformly bounded in $(\lambda, x) \in \mathbb{D}_{2 \varepsilon^{\prime}} \times \bar{P}_{r_{0}}$.

Thus, there exists $C=C_{m}>0$ such that whenever $|\lambda|<\varepsilon^{\prime}$ and $n \geq 0$,

$$
\begin{gathered}
\left|h_{\lambda, m}^{n}\left(c_{1}\right)-c_{1}-\lambda\right| \leq C|\lambda|^{m+1}, \\
\left|h_{\lambda, m}^{n}\left(a_{1}\right)-\varphi_{m}(\lambda)-a_{1}\right| \leq C|\lambda|^{m+1} .
\end{gathered}
$$

By Lemma 6.1, enlarging $C$ if necessary, we have

$$
\left|\varphi(\lambda)-\varphi_{m}(\lambda)\right| \leq C|\lambda|^{m / 3} \leq C|\lambda|^{m_{1}+1} .
$$

Put

$$
\mathcal{G}_{\lambda}^{(n)}(z)=G_{h_{\lambda, m}^{n}\left(c_{1}\right)} \circ G_{h_{\lambda, m}^{n+1}\left(c_{1}\right)} \circ \cdots \circ G_{h_{\lambda, m}^{n+q-1}\left(c_{1}\right)}(z) .
$$


Then $h_{\lambda, m}^{(n)}\left(g^{q}(z)\right)=\mathcal{G}_{\lambda}^{(n)}\left(h_{\lambda, m}^{(n+q)}(z)\right)$ and the sequence $\left\{\mathcal{G}_{\lambda}^{(n)}(z)\right\}$ is uniformly bounded in $\lambda \in \mathbb{D}_{\mathcal{\varepsilon}^{\prime}}, z \in B\left(a_{1}, \delta_{0}\right)$ for some $\delta_{0}>0$. By the lifting property (6.4), 6.5), (6.6) enlarging $C$ further, we have

$$
\left|D\left(\mathcal{G}_{\lambda}^{(n)}\right)\left(h_{\lambda, m}^{n+q}\left(a_{1}\right)\right)-\sigma(\lambda)\right| \leq C|\lambda|^{m_{1}+1} .
$$

for each $n \geq 0$. It follows that there exists $\varepsilon^{\prime \prime}>0$ such that whenever $|\lambda| \leq \varepsilon^{\prime \prime}$, we have

$$
\left|D \mathcal{G}_{\lambda}^{(n)}\left(h_{\lambda, m}^{n+q}\left(a_{1}\right)\right)-\left(\sigma(0)+3 A \lambda^{m_{1}}\right)\right|=O\left(|\lambda|^{m_{1}+1}\right) .
$$

Therefore, we can choose $\lambda \neq 0$ with $|\lambda|$ arbitrarily small and such that

$$
\left|D \mathcal{G}_{\lambda}^{(n)}\left(h_{\lambda, m}^{n+q}\left(a_{1}\right)\right)\right|<|\sigma(0)|-2|A||\lambda|^{m_{1}}<|\sigma(0)|=|\kappa|
$$

holds for every $n$. We fix such a choice of $\lambda$ now. $\delta$,

Let $\delta>0$ be a small constant such that for each $z$ and each $n \geq 0$ with $\left|z-h_{\lambda, m}^{n+q}\left(a_{1}\right)\right|<$

$$
\left|D \mathcal{G}_{\lambda}^{(n)}(z)\right|<|\sigma(0)|-|A||\lambda|^{m_{1}}=: \kappa^{\prime}<|\sigma(0)|=|\kappa| .
$$

Let $l_{0}>0$ be large enough such that for each positive integer $l \geq l_{0}$ and any $n \geq 0$, $\left|h_{\lambda, m}^{n}\left(a_{1}\right)-h_{\lambda, m}^{n}\left(c_{l q+1}\right)\right|<\delta$. Then for any $l \geq l_{0}$, and any $n \geq 0$,

$$
\begin{aligned}
\left|h_{\lambda, m}^{n}\left(a_{1}\right)-h_{\lambda, m}^{n}\left(c_{(l+1) q+1}\right)\right|=\mid \mathcal{G}_{\lambda}^{(n)}\left(h_{\lambda, m}^{n+q}\left(a_{1}\right)\right)-\mathcal{G}_{\lambda}^{(n)}\left(h_{\lambda, m}^{n+q}\left(c_{l q+1}\right) \mid\right. & \\
& \leq \kappa^{\prime}\left|h_{\lambda, m}^{n+q}\left(a_{1}\right)-h_{\lambda, m}^{n q}\left(c_{l q+1}\right)\right| .
\end{aligned}
$$

It follows that

$$
\left|h_{\lambda, m}^{0}\left(a_{1}\right)-h_{\lambda, m}^{0}\left(c_{l q+1}\right)\right|=O\left(\kappa^{\prime l}\right) .
$$

Let us now distinguish two cases to complete the proof by deducing a contradiction.

Case 1. $|\kappa|<1$. In this case, $P_{r_{0}}$ contains a neighborhood of $a_{1}$, so by the admissible property of $h_{\lambda, m}$, we have $\mid h_{\lambda, m}^{0}\left(a_{1}\right)-h_{\lambda, m}^{0}\left(c_{l q+1}|\asymp| a_{1}-\left.c_{l q+1}|\asymp| \kappa\right|^{l}\right.$. This leads to $|\kappa|<\left|\kappa^{\prime}\right|$, a contradiction!

Case 2. $|\kappa|=1$. In this case, $c_{l q+1}$ converges to $a_{1}$ only polynomially fast. However, since $h_{\lambda, m}$ is qc and hence bi-Hölder, (6.7) implies that $c_{l q+1}$ converges to $a_{1}$ exponentially fast, a contradiction!

\section{Applications to transversality FOR COMPlex families}

In this section we will show that Theorems 2.1 and 2.2 follow from the Main Theorem. First we show that the attracting periodic cycle contains the singular value in its immediate basin of attraction.

Let $\mathbb{R}_{+}=(0, \infty)$.

Proposition 7.1. Consider one of the following three situations:

(i) $g(z)=f(z)+c_{1}$ with $f \in \mathcal{F}, c_{1} \in \bar{D}$, and $\mathcal{O}$ is an attracting or parabolic cycle of $g$ with multiplier $\kappa \neq 0$;

(ii) $g(z)=c_{1} f(z)$ with $f \in \mathcal{E}, c_{1} \in D^{+} \backslash\{0\}$ and $\mathcal{O} \subset D \backslash\{0\}$ is an attracting or parabolic cycle of $g$ with multiplier $\kappa \neq 0$;

(iii) $g(z)=c_{1} f(z)$ with $f \in \mathcal{E}_{o}, c_{1} \in D^{+} \backslash\{0\}$ and $\mathcal{O} \subset D \backslash\{0\}$ is an attracting or parabolic cycle of $g$ with multiplier $\kappa \neq 0$ but $\mathcal{O} \neq-\mathcal{O}$;

Then

(1) there is a simply connected open set $W$ with the following property:

- $g^{k q}$ is univalent on $W$ for each $k \geq 1$,

- $g^{k q}$ converges uniformly on $W$ to a point in $\mathcal{O}$;

- $W \ni c_{1}$ in cases (i) and (ii), while $W \ni c_{i}$ or $W \ni-c_{i}$ in case (iii).

(2) if $\mathcal{O}$ is a parabolic periodic orbit then it is non-degenerate. 
Proof. We shall prove this proposition by the classical Fatou argument. Let $E=\left\{c_{1}\right\}$ in case (i) and (ii) and let $E=\left\{ \pm c_{1}\right\}$ in case (iii).

Note that the assumption implies that $0 \notin \mathcal{O}$ in all cases. Take a Jordan disk $A_{0} \subset$ $D \backslash\{0\}$ along with a univalent map $\varphi: A_{0} \rightarrow \mathbb{C}$ (Koenigs or Fatou coordinate) so that $g^{q^{\prime}}\left(A_{0}\right) \subset A_{0}$ where (i) in attracting case: $a_{0} \in A_{0}, q^{\prime}=q, \varphi\left(A_{0}\right)=\mathbb{D}_{\rho}$ for some $\rho>0$ and $\varphi \circ g^{q}=\kappa \varphi$ on $A_{0}$, (ii) in the parabolic case: $a_{0} \in \partial A_{0}, q^{\prime}=r q$ for a minimal $r \geq 1$, $\varphi\left(A_{0}\right)=\{z: \Re(z)>M\}$ for some $M>0$ and $\varphi \circ g^{q^{\prime}}=\varphi+1$ on $A_{0}$.

In each case, we observe that for any connected open set $B \subset D \backslash(E \cup\{0\}), g$ : $g^{-1}(B) \rightarrow B$ is an un-branched covering and $g^{-1}(B) \subset D \backslash\{0\}$. Here we use that in case (ii), (iii) that $c_{1} \in D_{+}$since this implies by assumption (c) in the definition of $\mathcal{E}, \mathcal{E}_{o}$, that $D / c_{1} \subset V$.

Let $A_{n}, n=1,2, \ldots$, denote the component of $g^{-n}\left(A_{0}\right)$ with $A_{q^{\prime} k} \supset A_{0}$ for each $k=$ $1,2, \ldots$ and $g\left(A_{n}\right) \subset A_{n-1}$. There exists a minimal $N \geq 0$ such that $A_{N} \cap E \neq \emptyset$. Indeed, otherwise, since $A_{0}$ is a simply connected open set contained in $D \backslash(E \cup\{0\})$, we obtain from the observation above by induction that $A_{n} \subset D \backslash(E \cup\{0\})$ and $g: A_{n} \rightarrow A_{n-1}$ is an un-branched covering for each $n \geq 1$. It follows that $g^{k q^{\prime}}: A_{k q^{\prime}} \rightarrow A_{0}$ is an un-branched covering, hence a conformal map for each $k=1,2, \ldots$. Thus $\varphi$ extends to a univalent function from $A=\bigcup_{k=0}^{\infty} A_{k q}$ onto $\mathbb{C}$ via the functional equation $\varphi\left(f^{q^{\prime}}(z)\right)=\kappa \varphi(z)$ or $\varphi\left(f^{q^{\prime}}(z)\right)=\varphi(z)+1$, which implies that $A=\mathbb{C}$, contradicting with $E \cap A=\emptyset$. Taking $W=A_{N}$ completing the proof of (1).

Let us now prove (2). So assume that $\mathcal{O}$ is parabolic. In case (i) and (ii), as the argument above shows that each attracting petal around $\mathcal{O}$ intersects the orbit of $c_{1}$, the cycle is non-degenerate. Assume now that we are in case (iii). Then either $c_{1}$ or $-c_{1} \in W$. Since the map $g$ is odd and $\mathcal{O} \neq-\mathcal{O}$, only one of $c_{1}$ and $-c_{1}$ is contained in the basin of attraction of $\mathcal{O}$, and thus the statements (2) hold for the same reason as before.

Proof of Theorems 2.1 and 2.2. The proposition above implies that $g$ (restricted to a suitable small domain $U$ ) is a marked map w.r.t. $c_{1}$. Choosing $r_{0}>0$ small enough, we have $P_{r_{0}}$ is compact subset of $U$. Then, as is in shown in [24, 25] the lifting property 2.2 holds. Indeed, let $h_{\lambda}^{(0)}:=h_{\lambda}: P_{r_{0}} \rightarrow \mathbb{C}, \lambda \in \mathbb{D}_{\epsilon}$ be a holomorphic motion. As is shown in [24, 25], one can define a sequence of holomorphic motions $h_{\lambda}^{(n)}$ as in (2) of Definition 2.2 so that properties (1),(3) also hold. So $(g, G)_{W}$ has the lifting property for the set $P_{r_{0}}$. In particular, the first parts of Theorems 2.1 2.2 follow from the Main Theorem.

\section{Application to families of ReAl maps}

In this section, we shall prove Theorem 2.3. To this end, we shall need the result in 24, 25. to determine the sign of $\kappa^{\prime}$ and $Q$ in the transversality inequalities in Theorems 2.1 and 2.2 .

Throughout let $f_{t}$ be a family as in the assumption of Theorem 2.3, case (i) or (ii). The case (iii) can be easily reduced to case (i). Put $c=0$ in case (i), so that $c$ is the common turning point of $f_{t}$. For $t<c, f_{t}$ has no periodic point of period greater than one, so in the following, we shall mainly concerned with

$$
t \in J_{+}:=(c, \infty) \cap J .
$$

The following is an immediate consequence of Proposition 7.1.

Proposition 8.1. Suppose that $f_{c_{1}}, c_{1} \in J^{+}$, has an attracting or parabolic cycle $\mathcal{O} \subset J$ and let $a_{0}$ be the rightmost point in $\mathcal{O}$. Then $f^{k q}$ is monotone on $\left[a_{0}, c_{1}\right]$ for all $k \geq 0$ and $f^{k} q$ converges uniformly on $\left[a_{0}, c_{1}\right]$ to $a_{0}$ as $k \rightarrow \infty$.

Proof. Let $\tilde{f}$ denote the complex extension in $\mathcal{F}$ or $\mathcal{E} \cup \mathcal{E}_{o}$. Since $\tilde{f}$ is real symmetric, the simply connected domain $W$ as claimed in Proposition 7.1 can be taken to be symmetric with respect to $\mathbb{R}$. If $c_{1} \in W$, then the statement follows. If $c_{1} \notin W$, then $f \in \mathcal{E}_{o, u}$, $-c_{1} \in W$ and there exists $a_{0} \in \mathcal{O}$ such that $f^{k q}$ converges to $a_{0}$ on the interval $K$ 
bounded by $a_{0}$ and $-c_{1}$. However, $a_{0}>0$ and $-c_{1}<0$ so $K \ni 0$. Since $f(0)=0$, this is absurd!

Proposition 8.2. If $g:=f_{c_{1}}$ has a cycle $\mathcal{O}$ with multiplier 0 , and let $\kappa(t)$ denote the multiplier of the attracting cycle of $f_{t}$ for $t$ close to $c_{1}$. Then there exists $\varepsilon>0$ such that $\kappa(t)>0$ for $t \in\left(c_{1}-\varepsilon, c_{1}\right)$ and $\kappa(t)<0$ for $t \in\left(c_{1}, c_{1}+\varepsilon\right)$.

Proof. In [24], 25] the following inequality was proved

$$
\frac{\left.\frac{d}{d t} f_{t}^{q}(c)\right|_{t=c_{1}}}{D g^{q-1}\left(c_{1}\right)}>0
$$

Let us deduce the conclusion of the proposition. Let $a(t)$ denote the fixed point of $f_{t}^{q}$ near $c$ for $t$ close to $c_{1}$. Assume for definiteness that $D g^{q-1}\left(c_{1}\right)>0$. Then it follows that there is $\varepsilon>0$ such that $f_{t}^{q}(c)>c$ for $t \in\left(c_{1}, c_{1}+\varepsilon\right)$ and $f_{t}^{q}(c)<c$ for $t \in\left(c_{1}-\varepsilon, c_{1}\right)$. Thus for $t \in\left(c_{1}, c_{1}+\varepsilon\right), a(t)>c$ and for $t \in\left(c_{1}-\varepsilon, c_{1}\right), a(t)<c$. Reducing $\varepsilon$ if necessary, $D g_{t}^{q-1}\left(g_{t}(a(t))>0\right.$, so $\kappa(t)=D g(a(t)) D g^{q-1}\left(g_{t}(a(t))\right.$ has the sign as claimed.

We say an open subinterval $J_{1}$ of $J$ is an attracting window if for each $t \in J_{1}, f_{t}$ has an attracting periodic cycle $\mathcal{O}_{t}$ with multiplier $\kappa(t) \in(-1,1)$. By the Implicit Function Theorem, $\mathcal{O}_{t}$ and $\kappa(t)$ depending on $t$ in a $C^{1}$ way. In particular, the cycles $\mathcal{O}_{t}$ have the same period, which is called the period of the attracting window.

Lemma 8.3. Let $J_{1}$ be an attracting window of period $q \geq 2$ and let $\kappa(t)$ be the corresponding multiplier function. Then $\kappa(t)$ is strictly decreasing in $J_{1}$.

Proof. Assume without loss of generality that $J_{1}=\left(t_{-}, t_{+}\right)$is a maximal attracting window. First, by Lemma 8.2 , $\kappa$ can have at most one zero in $J_{1}$. Indeed, otherwise, let $c_{1}<\hat{c}_{1}$ be two consecutive zeros, then it follows that $\kappa<0$ in a right neighborhood of $c_{1}$ and $\kappa>0$ in a left neighborhood of $\hat{c}_{1}$, which implies by the intermediate value theorem that there is another zero of $\kappa$ in-between $c_{1}$ and $\hat{c}_{1}$, absurd!

Now let us assume that $\kappa\left(t_{0}\right)=0$ for some $t_{0} \in J_{1}$. Then $\kappa(t)>0$ for $t \in\left(t_{-}, t_{0}\right)$ and $\kappa(t)<0$ for $t \in\left(t_{0}, t_{+}\right)$. By Theorems 2.1 and 2.2, $\kappa^{\prime}(t) \neq 0$ for each $t \neq t_{0}$. This forces $\kappa^{\prime}(t)<0$ for all $t \in J_{1} \backslash\{0\}$, so $\kappa(t)$ is strictly decreasing in $J_{1}$.

Finally, let us prove that $\kappa$ does have a zero in $J_{1}$. Indeed, it is easy to see $t_{-}, t_{+} \in J$, so by the maximality of $t_{+}$, we have $\kappa(t) \rightarrow \pm 1$ as $t \nearrow t_{ \pm}$. Since $\kappa$ is monotone in $J_{1}$ (by Theorems 2.1 and 2.2 , the intermediate value theorem implies that $\kappa$ has a zero.

Proposition 8.4. Assume that for some $c_{1} \in J_{+}, g=f_{c_{1}}$ has a parabolic cycle with multiplier 1. Let $q$ be the period of the cycle and let $a_{0}$ be the rightmost point in the cycle. Then there exist $\varepsilon>0$ and $\delta>0$ such that for each $t \in\left(c_{1}, c_{1}+\varepsilon\right), f_{t}$ has an attracting cycle of period $q$ and $a_{0}(t) \rightarrow a_{0}$ as $t \rightarrow c_{1}$, and for each $t \in\left(c_{1}-\varepsilon, c_{1}\right)$ and $x \in\left[a_{0}-\delta, a_{0}+\delta\right], f_{t}^{q}(x)<x$. Equivalently, for $a_{0}:=\lim _{k \rightarrow \infty} g^{k q}\left(c_{1}\right)$, we have

$$
Q\left(a_{0}\right):=\left.\frac{d}{d t} f_{t}^{q}\left(a_{0}\right)\right|_{t=c_{1}}>0 .
$$

Proof. By Proposition 8.1, $c_{k q+1}$ decreases to $a_{0}$ and $D^{2} g^{q}\left(a_{0}\right)<0$. By Theorems 2.1 and 2.2 $Q\left(a_{0}\right) \neq 0$. So $f_{t}^{q}(x)$ displays a saddle-node bifurcation at $\left(a_{0}, c_{1}\right)$. It is wellknown, see for example [6. Proposition 7.7.5] that there exist $\varepsilon>0$ and $\delta>0$ such that for each $t$ in

$$
J_{1}= \begin{cases}{\left[c_{1}, c_{1}+\varepsilon\right)} & \text { if } Q\left(a_{0}\right)>0, \\ \left(c_{1}-\varepsilon, c_{1}\right] & \text { if } Q\left(a_{0}\right)<0,\end{cases}
$$

$f_{t}^{q}$ has two fixed points $a_{-}(t)$ and $a_{+}(t)$, depending continuously in $t$, with $a_{-}\left(c_{1}\right)=$ $a_{+}\left(c_{1}\right)=a_{0}$, and with $0<D f_{t}^{q}\left(a_{-}(t)\right)<1$ and $D f_{t}^{q}\left(a_{+}(t)\right)>1$, while for $t$ in

$$
J_{2}= \begin{cases}\left(c_{1}-\varepsilon, c_{1}\right) & \text { if } Q\left(a_{0}\right)>0, \\ \left(c_{1}, c_{1}+\varepsilon\right) & \text { if } Q\left(a_{0}\right)<0,\end{cases}
$$


$f_{t}^{q}(x)<x$ for each $\left|x-a_{0}\right| \leq \delta$. So $J_{1}^{o}$ is an attracting window, and by Lemma 8.3 , the multiplier function $\kappa(t)=D f_{t}^{q}\left(a_{-}(t)\right)$ is monotone decreasing. Thus $J_{1}=\left[c_{1}, c_{1}+\varepsilon\right)$ and $Q\left(a_{0}\right)>0$.

Proposition 8.5. Assume that $f_{c_{1}}$ has a parabolic cycle $\mathcal{O}$ of period $q$ and with multiplier -1 . Then for any positive integer $N \geq 1$, there exist $\varepsilon>0$ and $\delta>0$ such that for each $t \in\left(c_{1}-\varepsilon, c_{1}+\varepsilon\right), f_{t}^{2 N q}$ has exactly three fixed points in the $\delta$-neighborhood of $\mathcal{O}$, two of them hyperbolic attracting, and one hyperbolic repelling.

Proof. Note that the assumption implies that $c_{1} \in J_{+}$. For the case $N=1$, this is a well-known fact about periodic doubling bifurcation. Indeed, by Theorems 2.1 and 2.2 , $\kappa^{\prime}\left(c_{1}\right) \neq 0$, where $\kappa(t)$ is the multiplier of the periodic orbit of $f_{t}$ of period $q$ near $\mathcal{O}$ for $t$ close to $c_{1}$. We must have $\kappa^{\prime}\left(c_{1}\right)<0$ for otherwise, a small right-sided neighborhood of $c_{1}$ would be an attracting window on which the multiplier function is increasing which is ruled out by Lemma 8.3. On the other hand, by Proposition 7.1, $D^{3} g^{2 q}(a) \neq 0$ for each $a \in \mathcal{O}$. Thus the statement follows, for example, by [6, Proposition 7.7.6].

The case for general $N$ follows: $D^{3} g^{2 q N}(a) \neq 0$ for $a \in \mathcal{O}$. For reducing $\varepsilon, \delta$ if necessary, $f^{2 N q}$ has at most three fixed points in the $\delta$-neighborhood of $\mathcal{O}$ for $t \in\left(c_{1}-\varepsilon, c_{1}\right)$.

Proof of Theorem 2.3. Let $\kappa$ be the multiplier the $f_{t_{*}}$-cycle $\mathcal{O}$. First let us assume $\kappa \neq 1$. Then by the Implicit Function Theorem, there is a maximal $T$ such that $J_{1}:=\left[c_{1}, T\right) \subset J$ and each $f_{t}, t \in J_{1}$, has a periodic cycle $\mathcal{O}_{t}$ which depends on $t$ continuously and such that $\mathcal{O}_{t}=\mathcal{O}$ and the multiplier $\kappa(t) \neq 1$. We shall prove that $T$ is the right endpoint of $J$. Arguing by contradiction, assume that this is not the case. By the maximality of $T, \lim _{t \rightarrow T} \kappa(t)=1$. By the intermediate value theorem, we are in one of the following cases:

Case 1. $\kappa(t)>1$ for all $t \in J_{1}$. Choose a subsequence $t_{n} \nearrow T$ such that $\mathcal{O}_{t_{n}}$ converges to a periodic orbit $\mathcal{O}_{T}$ of $f_{T}$. Let $q$ denote the period of $\mathcal{O}$ and $q^{\prime}$ the period of $\mathcal{O}_{T}$, then $q=q^{\prime} m$ for some positive integer $m$.

If $\mathcal{O}_{T}$ has multiplier 1, then by Proposition 8.4, there exist $\varepsilon>0$ and $\delta>0$ such that $f_{t}^{q^{\prime}}(x)<x$ for each $t \in(T-\varepsilon, T)$ and $\left|x-a_{0}(T)\right|<\delta$, where $a_{0}(T)$ denotes the rightmost point in the parabolic cycle $\mathcal{O}_{T}$. By continuity, there exist $\varepsilon^{\prime}, \delta^{\prime}>0$, we have $f_{t}^{q}(x)<x$ when $t \in\left(T-\varepsilon^{\prime}, T\right)$ and $\left|x-a_{0}(T)\right|<\delta^{\prime}$. This is in contradiction with the construction of $\mathcal{O}_{T}$.

Assume now that $\mathcal{O}_{T}$ has multiplier -1 . Then $q=2 q^{\prime} N$ for some integer $N \geq 1$. By Proposition 8.5. for each $n$ large enough, $f_{t_{n}}^{q}$ has exactly three fixed points, two of which are attracting. However $\mathcal{O}_{t_{n}}$ contains at least two repelling fixed point of $f_{t_{n}}^{q}$, a contradiction!

Case 2. $\kappa(t)<1$ for all $t \in J_{1}$. Then there exists $\varepsilon>0$ such that $(T-\varepsilon, T)$ is an attracting window and by Lemma $8.3, \kappa(t)$ is strictly decreasing, thus contradicting the maximality of $T$.

So now let us consider the case $\kappa=1$. By Proposition 8.4, there is $\varepsilon>0$ such that $\left(c_{1}, c_{1}+\varepsilon\right)$ is an attracting window. By what have proved above, the attracting periodic orbits allow further continuation until the right endpoint of $J$.

Remark 8.6. Figure 1 shows the bifurcation diagram for the family $f_{c_{1}}(x)=c_{1} \sin (x)$, $c_{1} \in \mathbb{R}$. It also shows that for this family there are degenerate parabolic bifurcations, which occur when $\mathcal{O}=-\mathcal{O}$.

\section{Appendix A. In the REAL PARABOLIC CASE $\left.\frac{\partial G_{w}^{q}}{\partial w}\left(a_{0}\right)\right|_{w=c} \geq 0$ HOLDS}

We assume that $g$ is real marked map w.r.t. $c_{1}$ so that the sequence $c_{n}:=g^{n-1}\left(c_{1}\right)$, $n=1,2, \ldots$ tends to a non-degenerate parabolic periodic orbit $\mathcal{O}:=\left\{a_{0}, \ldots, a_{q-1}\right\}$ with multiplier +1 . Consider a holomorphic deformation $(g, G)_{W}$ and assume that $(g, G)_{W}$ has the lifting property for the set $P=\left\{c_{n}\right\}_{n \geq 1}$ (notice that this condition is local and 


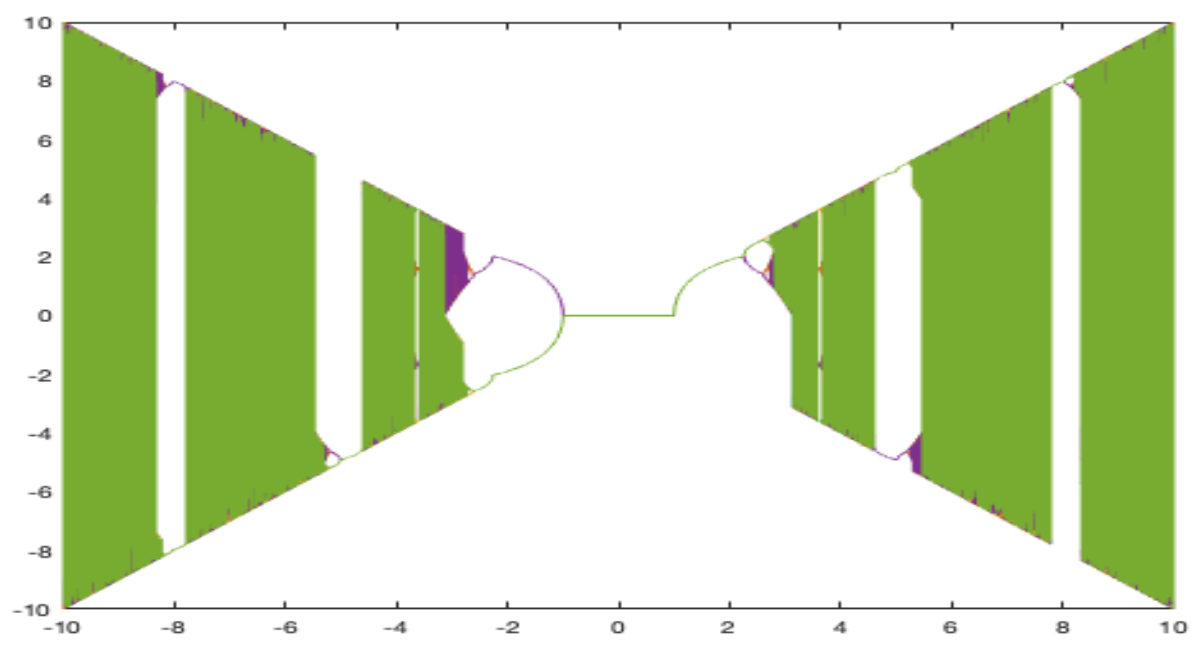

FIGURE 1. The bifurcation diagram of $f_{c}(x)=c \sin (x)$. For each $c \in[-10,10]$, the last 100 iterates from the set $\left\{f_{c}^{k}(\pi / 2)\right\}_{k=0}^{1000}$ are drawn (in the vertical direction). The bifurcation points for which only 'one half of a parabola' is visible, correspond to pitchfork bifurcations (where both critical values are attracted to a single parabolic periodic point), and where two halves are visible correspond to period doubling bifurcations. Note that $\sin \in \mathcal{E}_{o}$, where $D=D_{+}=V=\mathbb{C}$.

weaker than the one of the Main Theorem). Under these assumptions we will show that $Q\left(c_{1}\right)=\left.\frac{\partial G_{w}^{q}}{\partial w}\right|_{w=c_{1}}\left(a_{0}\right) \geq 0$.

This Appendix will also motivate the choice of the particular vector field along $P$ appearing in Section 4.

From (4.3) it follows that the 2nd equality holds in

$$
\Delta(z):=\sum_{j=1}^{q} \frac{L\left(g^{j-1}(z)\right)}{D g^{j}(z)}=\left.\frac{1}{D g^{q}(z)} \frac{\partial G_{w}^{q}}{\partial w}\right|_{w=c}(z)
$$

Since $D g^{q}\left(a_{0}\right)=1$,

$$
\Delta\left(a_{0}\right)=\left.\frac{\partial G_{w}^{q}}{\partial w}\right|_{w=c}\left(a_{0}\right)=\sum_{j=1}^{q} \frac{L\left(g^{j-1}\left(a_{0}\right)\right)}{D g^{j}\left(a_{0}\right)}=\sum_{j=0}^{q-1} \frac{L\left(g^{j}\left(a_{0}\right)\right)}{D g^{j+1}\left(a_{0}\right)} .
$$

Proposition A.1. Assume that $(g, G)_{W}$ has the lifting property for the set $P$. Moreover, assume that $g$ is real, has a periodic point $a_{0}$ of period $q$ with $D g^{q}\left(a_{0}\right)=1, D^{2} g^{q}\left(a_{0}\right) \neq 0$ so that $c_{1}$ is in the basin of $a_{0}$ and so that $D g^{q}\left(c_{k q+1}\right)>0$ for each $k \geq 0$. Then

$$
\left.\frac{\partial G_{w}^{q}}{\partial w}\right|_{w=c}\left(a_{0}\right) \geq 0 .
$$

Proof of Proposition A.1. By Proposition A.2, see also [20],

$$
D(\rho):=1+\sum_{n=1}^{\infty} \frac{\rho^{n} L\left(c_{n}\right)}{D g^{n}\left(c_{1}\right)}>0 \text { for all } 0<\rho<1 .
$$

Arguing by contradiction, assume that $\Delta\left(a_{0}\right)<0$. Then there exists $\rho_{0} \in(0,1)$ and $k_{0} \geq 1$ such that for each $\rho_{0}<\rho<1$ and each $k \geq k_{0}$, we have

$$
B_{k}(\rho):=\sum_{j=1}^{q} \frac{\rho^{j} L\left(c_{q k+j}\right)}{D g^{j}\left(c_{q k+1}\right)}<\Delta\left(a_{0}\right) / 2 .
$$

By assumption, $M_{k}:=k^{2} D g^{k q}\left(c_{1}\right)>0$ holds for all $k \geq 0$. By Leau-Fatou Flower theorem, see Appendix B,

$$
D g^{q}\left(c_{k q+1}\right)=1-2 / k+O\left(\log k k^{-2}\right),
$$


so $M:=\lim _{k \rightarrow \infty} M_{k}>0$ exists. Enlarging $k_{0}$, we have $M_{k}>M / 2$ for all $k \geq k_{0}$. Then

$$
\begin{aligned}
D(\rho) & =1+\sum_{n=1}^{\infty} \frac{\rho^{n} L\left(c_{n}\right)}{D g^{n}(c)}=1+\sum_{k=0}^{\infty} \sum_{j=1}^{q} \frac{\rho^{q k+j} L\left(c_{q k+j}\right)}{D g^{q k+j}(c)}= \\
& =1+\sum_{k=0}^{\infty}\left[\frac{\rho^{q k}}{D g^{q k}\left(c_{1}\right)} \sum_{j=1}^{q} \frac{\rho^{j} L\left(c_{q k+j}\right)}{D g^{j}\left(c_{q k+1}\right)}\right]=1+\sum_{k=0}^{\infty} \frac{k^{2} \rho^{q k}}{M_{k}} B_{k}(\rho) \\
& \leq 1+\sum_{k=0}^{k_{0}} \frac{k^{2} \rho^{q k}}{M_{k}} B_{k}(\rho)+\frac{\Delta\left(a_{0}\right)}{M} \sum_{k=k_{0}+1}^{\infty} k^{2} \rho^{q k},
\end{aligned}
$$

provided that $\rho_{0}<\rho<1$. Since $\sum_{k=k_{0}+1}^{\infty} k^{2} \rho^{q k} \rightarrow \infty$ as $\rho \rightarrow 1$, this implies that $\liminf _{\rho \nearrow 1} D(\rho)=-\infty$, a contradiction with $D(\rho)>0$ for all $\rho \in(0,1)$.

A.1. A vector field $v_{\rho}$ along $P$ so that $\rho \mathcal{A} v_{\rho}=v_{\rho}$.

Proposition A.2. Let $g$ be a marked map w.r.t. $c_{1}$, and $P=\left\{c_{n}\right\}_{n \geq 1}$ converges to the periodic orbit $\mathcal{O}$ of $g$ which has the multiplier +1 and not degenerate. Consider a holomorphic deformation $(g, G)_{W}$. Assume that $(g, G)_{W}$ has the lifting property for the set $P$. Then for all $|\rho|<1$ one has

$$
1+\sum_{n \geq 1} \frac{\rho^{n} L\left(c_{n}\right)}{D g^{n}\left(c_{1}\right)} \neq 0
$$

where $L(x)=\left.\frac{\partial G_{w}}{\partial w}\right|_{w=c_{1}}(x)$.

Proof. Let us for the moment assume that $h_{\lambda}\left(c_{i}\right)=c_{i}+v_{i} \lambda+O\left(\lambda^{2}\right)$ defines a holomorphic motion of $P$. Then its lift $\hat{h}_{\lambda}\left(c_{i}\right)=c_{i}+\hat{v}_{i} \lambda+O\left(\lambda^{2}\right)$ is defined for $|\lambda|$ small and

$$
G_{\left.h_{\lambda}\left(c_{1}\right)\right)}\left(\hat{h}_{\lambda}\left(c_{i}\right)\right)=h_{\lambda}\left(c_{i+1}\right)=c_{i+1}+v_{i+1} \lambda+O\left(\lambda^{2}\right) .
$$

Writing $D_{i}=D g\left(c_{i}\right)$ we obtain

$$
L_{i} v_{1}+D_{i} \hat{v}_{i}=v_{i+1}, i \geq 1
$$

where $L_{i}=L\left(c_{i}\right)$. Taking $v=\left(v_{1}, v_{2}, \ldots\right)$ and $\hat{v}=\left(\hat{v}_{1}, \hat{v}_{2}, \ldots\right)$ we have that $\hat{v}=\mathcal{A} v$ where

$$
\mathcal{A}=\left(\begin{array}{cccccc}
-L_{1} / D_{1} & 1 / D_{1} & 0 & \ldots & \ldots & \ldots \\
-L_{2} / D_{2} & 0 & 1 / D_{2} & 0 & \ldots & \ldots \\
-L_{3} / D_{3} & 0 & 0 & 1 / D_{3} & \ldots & \ldots \\
\vdots & \vdots & \vdots & \vdots & \ddots & \vdots
\end{array}\right)
$$

Assume $\rho \neq 0$ and consider the vector field $v_{\rho}$ on $P$ defined for $n>1$ by

$$
\begin{aligned}
v_{\rho}\left(c_{n}\right) & :=\frac{D g^{n-1}\left(c_{1}\right)}{\rho^{n-1}} \sum_{k=0}^{n-1} \frac{\rho^{k} L_{k}}{D g^{k}\left(c_{1}\right)}= \\
& =L_{n-1}+\frac{D g\left(c_{n-1}\right) L_{n-2}}{\rho}+\frac{D g^{2}\left(c_{n-2}\right) L_{n-3}}{\rho^{2}}+\cdots+\frac{D g^{n-1}\left(c_{1}\right) L_{0}}{\rho^{n-1}}
\end{aligned}
$$

(with $L_{0}=1$ ) and $v_{\rho}\left(c_{1}\right)=1$. Notice that for this vector field we get $\rho \mathcal{A} v_{\rho}=v_{\rho}$.

Assume, by contradiction, that for some $0<|\rho|<1$,

$$
1+\sum_{n \geq 1} \frac{\rho^{n} L\left(c_{n}\right)}{D g^{n}\left(c_{1}\right)}=0 .
$$


Then we have that

$$
v_{\rho}\left(c_{n}\right)=-\frac{D g^{n-1}(c)}{\rho^{n-1}} \sum_{k=n}^{\infty} \frac{\rho^{k} L\left(c_{k}\right)}{D g^{k}(c)}=-\sum_{j=1}^{\infty} \frac{\rho^{j} L\left(c_{n+j-1}\right)}{D g^{j}\left(c_{n}\right)} .
$$

For simplicity write $v_{i, \rho}=v_{\rho}\left(c_{i}\right)$. In the next lemma we will show that $v_{\rho}$ defines a Lipschitz vector field. Because of this, $v_{\rho}$ defines a holomorphic motion $h_{\lambda, \rho}$ for $|\lambda|<\epsilon$ for some $\epsilon>0$. As $\rho$ is fixed, let us write $h_{\lambda}=h_{\lambda, \rho}$. Since $(g, G)_{W}$ has the lifting property, it follows that the consecutive sequence of lifts $h_{\lambda}^{(n)}$ of $h_{\lambda}$ form a normal family. Write $h_{\lambda}^{(n)}(x)=x+\lambda v_{\rho}^{(n)}(x)+O\left(\lambda^{2}\right)$. Then $v_{\rho}^{(n)}=\frac{1}{\rho^{n}} v_{\rho}$ which, since $\rho<1$, contradicts that the family $h_{\lambda}^{(n)}$ forms a normal family.

Lemma A.3. The vector field

$$
v_{\rho}\left(c_{n}\right):=\sum_{j=1}^{\infty} \frac{\rho^{j} L\left(c_{n+j-1}\right)}{D g^{j}\left(c_{n}\right)}
$$

defined on the set $P=\left\{c_{i}\right\}_{i \geq 1}$ is Lipschitz.

Proof. Given $x \in U$ such that $g^{i}(x) \in U$ for all $i \geq 1$, define

$$
V_{\rho}(x)=\sum_{j=1}^{\infty} \frac{\rho^{j} L\left(g^{j-1}(x)\right)}{D g^{j}(x)} .
$$

We have $V_{\rho}\left(c_{n}\right)=v_{\rho}\left(c_{n}\right), n=1,2, \cdots$. Moreover,

$$
V_{\rho}^{\prime}(x)=\sum_{j=1}^{\infty} \rho^{j}\left[\frac{L^{\prime}\left(g^{j-1}(x)\right)}{D g\left(g^{j-1}(x)\right.}-\frac{L\left(g^{j-1}(x)\right)}{D g^{j}(x)} \sum_{i=0}^{n-1} \frac{D^{2} g\left(g^{i}(x)\right)}{D g\left(g^{i}(x)\right)}\right] .
$$

Since the periodic orbit $\mathcal{O}=\left\{a_{0}, \cdots, a_{q-1}\right\}$ of $g: U \rightarrow \mathbb{C}$ has multiplier +1 and is not degenerate, by the proof of Lemma B.1 for each $a_{j}$ there is a convex set $\Delta_{j}$ in the basin of $\mathcal{O}$ with a boundary point $a_{j}$ such that $g^{q}\left(\Delta_{j}\right) \subset \Delta_{j}$. Moreover, the closures of $\Delta_{j}, 0 \leq j \leq q-1$ are pairwise disjoint, all but finitely many points of the orbit $P$ are in $\Delta:=\cup_{j=0}^{q-1} \Delta_{j}$. Since $g^{n q}$ converges uniformly on $\Delta_{j}$ to $a_{j}$, it follows that $D g^{j}(x) \leq C \rho^{-j / 2}$, where $C$ is a constant. These bounds along with the definition for $V_{\rho}$, (A.3) and since $|\rho|<1$ imply that for some $K>0$ and all $x \in \Delta$,

$$
\left|V_{\rho}(x)\right| \leq K, \quad\left|V_{\rho}^{\prime}(x)\right| \leq K .
$$

As each component $\Delta_{j}$ of $\Delta$ is convex (so that $x_{1}, x_{2} \in \Delta_{j}$ implies $\left|V_{\rho}\left(x_{1}\right)-V_{\rho}\left(x_{2}\right)\right| \leq$ $\left.K\left|x_{1}-x_{2}\right|\right)$ and only finitely many points of $P$ is outside of $\Delta$ we conclude that $V_{\rho}$ is Lipschitz on $P$.

\section{Appendix B. The Leau-Fatou Flower}

Suppose that $\mathcal{O}$ is a non-degenerate parabolic periodic orbit as above. Fix $\alpha \in(0,1)$. For each $j$, and $r>0$, define

$$
\begin{gathered}
\Theta_{j}^{\text {att }}=\left\{\theta \in[0,1): D^{p+1} g^{p q}\left(a_{j}\right) e^{2 \pi i p \theta} \text { is real and negative }\right\}, \\
\Theta_{j}^{\text {rep }}=\left\{\theta \in[0,1): D^{p+1} g^{p q}\left(a_{j}\right) e^{2 \pi i p \theta} \text { is real and positive }\right\}, \\
\mathcal{C}_{j}(r)=\left\{a_{j}+s e^{2 \pi i t}: 0<s<r,|t-\theta|<s^{\alpha} \text { for some } \theta \in \Theta_{j}^{\text {rep }}\right\},
\end{gathered}
$$

and

$$
\mathcal{C}_{j}^{\prime}(r)=\left\{a_{j}+s e^{2 \pi i t}: 0<s<r,|t-\theta|<s^{\alpha} \text { for some } \theta \in \Theta_{j}^{\text {attr }}\right\},
$$

The following is a variation of the well-known Leau-Fatou Flower Theorem.

Lemma B.1 (Leau-Fatou Flower Theorem). (1) For each $r>0$, there exists $\tau=$ $\tau(r)>0$ such that

$$
B\left(a_{j}, \tau\right) \backslash \Omega_{r} \subset \mathcal{C}_{j}(\tau) .
$$


(2) For any $r>\tau>0$ and $z_{0} \in \Omega_{r}^{o}$, there exists $n_{0}=n_{0}\left(z_{0}\right)$ such that

$$
g^{n}\left(z_{0}\right) \in \bigcup_{j} \mathcal{C}_{j}^{\prime}(\tau)
$$

for all $n \geq n_{0}$.

Proof. This result is essentially contained in [8] or [32, so we will contend ourself with a sketch in the case that $\mathcal{O}=\{0\}$ and $g(z)=z-z^{p+1}+O\left(z^{p+2}\right)$. So $\Theta^{a t t r}=\{2 \pi j / p$ : $0 \leq j<p\}$ and $\Theta^{r e p}=\{\pi(2 j+1) / p: 0 \leq j<p\}$.

Let us first prove (2). As described in [32] there are $p$ attracting petals $U_{j}, 0 \leq j<p$, such that $U_{j}$ lies in the sector $(2 j-1) \pi / p<\theta<(2 j+1) \pi / p$ and such that for each $z_{0} \in U$ with $z_{n}:=g^{n}\left(z_{0}\right) \rightarrow 0, z_{n} \neq 0$, there exists $n_{0}$ such that $z_{n} \in U_{j}$ for some $j$ and all $n \geq 0$. Let us prove that $z_{n}$ eventually lands in $\mathcal{C}_{j}^{\prime}$. Indeed, assuming $j=0$ without loss of generality, and putting $w_{n}=-z_{n}^{-p}$, we have

$$
w_{n+1}=w_{n}+1+O\left(\left|w_{n}\right|^{-1 / p}\right) .
$$

From this, it is easy to check that for any $\tau>0, z_{n} \in \mathcal{C}^{\prime}(\tau)$ for all $n \geq n(\tau)$. The statement (2) is proved.

Let us prove the statement (1). First, by [32], there exists $r_{*}>0$ such that if $\left\{w_{-n}\right\}_{n=0}^{\infty}$ is a $g$-backward orbit inside $\overline{B\left(0, r_{*}\right)}$, then $w_{-n} \rightarrow 0$. We may assume without loss of generality that $r \in\left(0, r_{*}\right)$. Next, we check that there exists $\tau_{0}>0$ such that for any $\tau \in\left(0, \tau_{0}\right)$ the set $\mathcal{C}(\tau)$ is backward invariant under $g: g \mid \mathcal{C}(\tau)$ is injective and $g(\mathcal{C}(\tau) \supset \mathcal{C}(\tau)$. Arguing by contradiction, assume that the statement $(1)$ is false for some $r \in\left(0, r_{*}\right)$. Then for any $n \geq 1$, there is $z_{n} \in B(0,1 / n) \backslash(\mathcal{C}(1 / n) \cup\{0\})$ and a minimal positive integer $k_{n}$ such that $g^{k_{n}}\left(z_{n}\right) \notin B(0, r)$. Passing to a subsequence we may assume $g^{k_{n}-j}\left(z_{n}\right) \rightarrow w_{-j}$ as $n \rightarrow \infty$ for each $j$. Thus we obtain a $g$-backward orbit $\left\{w_{-j}\right\}_{j=1}^{\infty}$ with $w_{-j} \notin \mathcal{C}(\tau)$ and $w_{-j} \in \overline{B(0, r)} \backslash \mathcal{C}(\tau)$ for $j \geq 1$. However, applying the statement (2) to $g^{-1}$, we see that this is impossible.

\section{REFERENCES}

[1] L. Ahlfors. Lectures on quasiconformal mappings. Second edition. With supplemental chapters by C. J. Earle, I. Kra, M. Shishikura and J. H. Hubbard. University Lecture Series, 38. American Mathematical Society, Providence, RI, 2006.

[2] K. Astala,T. Iwaniec and G. Martin, Elliptic partial differential equations and quasiconformal mappings in the plane. Princeton Mathematical Series, 48. Princeton University Press, Princeton, NJ, 2009. xviii+677 pp. ISBN: 978-0-691-13777-3

[3] M. Astorg, Summability condition and rigidity for finite type maps, arXiv:1602.05172v1.

[4] A. Avila, M. Lyubich and W de Melo. Regular or stochastic dynamics in real analytic families of unimodal maps, Invent. Math. 154 (2003), no. 3, 451-550.

[5] L. Bers, H.L. Royden Holomorphic families of injections, Acta Math. 157 (1986), 259-286.

[6] M. Brin and G. Stuck. Introduction to Dynamical Systems. Cambridge University Press, 2004.

[7] X. Buff and A. Epstein, Bifurcation measure and postcritically finite rational maps, Complex dynamics, 491-512, A K Peters, Wellesley, MA, 2009.

[8] L. Carleson and T. Gamelin. Complex Dynamics. Springer-Verlag.

[9] A. Douady. Topological entropy of unimodal maps: monotonicity for quadratic polynomials. (Real and complex dynamical systems, Hillerod, 1993), 65-87. Kluwer, 1995.

[10] A. Douady and J.H. Hubbard, Itération des polynômes quadratiques complexes. C. R. Acad. Sci. Paris Sér. I Math. 294 (1982), no. 3, 123-126.

[11] A. Douady and J.H. Hubbard, Étude dynamique des polynômes complexes. Publications Mathématiques d'Orsay, Université de Paris-Sud, Département de Mathématiques, Orsay, 1984

[12] A. Douady and J.H. Hubbard, A proof of Thurston's topological characterization of rational functions. Acta Math. 171 (1993), no. 2, 263-297.

[13] A. Epstein. Towers of finite type complex analytic maps, PhD thesis, City University of New York, 1993.

[14] A. Epstein, Infinitesmimal Thurston rigidity and the Fatou-Shishikura inequality, Stony Brook IMS preprint $1999 \# 1$.

[15] A. Epstein, Transversality in holomorphic dynamics, http://homepages.warwick.ac.uk/ mases/ Transversality.pdf. 
[16] A. Epstein. https://icerm.brown.edu/materials/Slides/sp-s12-w1/Transversality_ Principles_in_Holomorphic_Dynamics_\%5D_Adam_Epstein,_University_of_Warwick.pdf

[17] C. Favre and T. Gauthier, Distribution of postcritically finite polynomials, Israel Journal of Mathematics 209 (2015), 235-292.

[18] G.M. Levin, On the theory of iterations of polynomial families in the complex plane. Translation from: Toeriya Funkzii, Funkzionalnyi Analiz i Ih Prilozheniya, No. 51 (1989), 94-106.

[19] G. M. Levin, Polynomial Julia sets and Pade's approximations (in Russian). Proceedings of XIII Workshop on Operator's Theory in Functional Spaces (Kyubishev, 6-13 October, 1988). Kyubishev State University, Kyubishev, 1988, 113-114

[20] G. Levin, On an analytic approach to the Fatou conjecture, Fund. Math. 171 (2002), no. 2, 177-196.

[21] G. Levin, On explicit connections between dynamical and parameter spaces, J. Anal. Math. 91 (2003), 297-327.

[22] G. Levin, Multipliers of periodic orbits in spaces of rational maps, Ergod. Th. \& Dynam. Sys. 31 (2011), 197-243.

[23] G. Levin, Perturbations of weakly expanding critical orbits. Frontiers in Complex Dynamics; In Celebration of John Milnor's 80th Birthday. Edited by Araceli Bonifant, Misha Lyubich and Scott Sutherland, Princeton Univ. Press, 2014, 163-196

[24] G. Levin, W. Shen and S. van Strien, Monotonicity of entropy and positively oriented transversality for families of interval maps. https://arxiv.org/abs/1611.10056

[25] G. Levin, W. Shen and S. van Strien, Positive Transversality via transfer operators and holomorphic motions with applications to monotonicity for interval maps. Nonlinearity 33 (2020), 3970-4012.

[26] G. Levin, W. Shen and S. van Strien, Transversality for critical relations of families rational maps: an elementary proof, in New Trends in One-dimensional Dynamics, Springer, Cham, 2019, pp. 201220.

[27] G. Levin, M.L. Sodin and P.M. Yuditski, A Ruelle operator for a real Julia set. Comm. Math. Phys. 141 (1991), no. 1, 119-132.

[28] P. Makienko, Remarks on Ruelle operator and invariant line field problem. II. Ergod. Th. \& Dynam. Sys. 25 (2005), 1561-1581.

[29] C.T. McMullen and D. Sullivan, Quasiconformal homeomorphisms and dynamics III: the Teichmüller space of a holomorphic dynamical system, Adv. Math. 135 (1998), 351-395.

[30] W. de Melo and S. van Strien, One-dimensional dynamics, Ergebnisse der Mathematik und ihrer Grenzgebiete. Springer-Verlag,1993, Berlin.

[31] J. Milnor, Periodic orbits, external rays and the Mandelbrot set, an expository account. Gómétrie complexe et systmes dynamiques (Orsay, 1995). Astérisque 261 (2000), xiii, 277-333.

[32] J. Milnor, Dynamics in one complex variable. Third Edition, Annals of Mathematics Studies 160, Princeton University Press, 2006.

[33] J. Milnor, Hyperbolic components. in Contemp. Math., 573, Conformal dynamics and hyperbolic geometry, 183-232, Amer. Math. Soc., Providence, RI, 2012.

[34] J. Milnor and W. Thurston. On iterated maps of the interval. Dynamical systems (College Park, MD, 1986-87), 465-563, Lecture Notes in Math., 1342, Springer, Berlin, 1988

[35] M. Rees. Components of degree two hyperbolic rational maps. Invent. Math. 100 (1990) 357-382.

[36] Z. Slodkowski, Holomorphic motions and polynomial hulls, Proc. Amer. Math. Soc. 111 (1991) 347-355.

[37] S. van Strien, Misiurewicz maps unfold generically (even if they are critically non-finite). Fund. Math. 163 (2000), no. 1, 39-54.

[38] D. Sullivan. Unpublished.

[39] M. Tsujii, A note on Milnor and Thurston's monotonicity theorem. Geometry and analysis in dynamical systems (Kyoto, 1993), 60-62, Adv. Ser. Dynam. Systems, 14, World Sci. Publ., River Edge, NJ, 1994.

[40] M. Tsujii, A simple proof of monotonicity of entropy in the quadratic family, Ergodic Theory Dynam. Systems 20 (2000), 925-933. 